\title{
Modelle, Techniken und Werkzeuge im Business Engineering
}

Robert Winter

1 Einführung

1.1 Methoden- und Modellbegriff

1.2 Modelltypen

2 Modellierungsebenen des Business Engineering

2.1 Verschiedene Ansätze zur Ebenenbildung

2.2 Modellierungsebenen im Business Engineering

3 Modelle und Techniken auf Strategieebene

3.1 Modellierung der Geschäftsarchitektur

3.2 Modellierung des Geschäftsmodells

3.3 Ableitung von Leistungen aus der Analyse von Kundenprozessen

4 Modelle und Techniken auf Prozessebene

4.1 Modellierung der Prozessarchitektur

4.2 Modellierung der Geschäftsprozesse

4.3 Modellierung der informationellen Aspekte

5 Modelle und Techniken auf Applikations- und Systemebene

5.1 Modellierung der Applikationsarchitektur

5.2 Applikationsmodellierung aus fachlicher Sicht

5.3 Modellierung von Komponenten

6 Werkzeuge

7 Schlussbetrachtung

8 Literatur 


\section{Einführung}

Business Engineering bezeichnet die methoden- und modellbasierte Konstruktionslehre für Unternehmen des Informationszeitalters [vgl. Beitrag von Österle/Winter in diesem Band]. Methoden und Modelle sowie Werkzeuge, welche die Methoden- und Modellanwendung unterstützen, spielen damit im Business Engineering eine zentrale Rolle. Die Nutzung von Methoden und Modellen schafft Transparenz, erzeugt eine Kommunikationsgrundlage, unterstützt die Arbeitsteiligkeit und dokumentiert den Prozess des Business Engineerings. Durch Methoden- und Modellnutzung unterscheidet sich arbeitsteiliges, ingenieurmässiges „Konstruieren“" von individualistischem „Schaffen“.

In diesem Beitrag werden in Abschnitt 1 grundlegende Begriffe geklärt. Die Ebenenstruktur des Business Engineering wird in Abschnitt 2 begründet. Die wichtigsten Modelle und Techniken des Business Engineerings auf Strategie-, Prozess- und Systemebene werden in den Abschnitten 3 bis 5 eingeführt. Abschnitt 6 diskutiert die Nutzung von Werkzeugen zur Unterstützung der Methoden- und Modellnutzung. Dieser Übersichtsbeitrag soll einen Überblick über die Methodik des Business Engineerings liefern. Der Schwerpunkt liegt auf der Einführung in ausgewählte Modelle und Techniken in ihrem Gesamtzusammenhang. Für eine detailliertere Darstellung bestimmter Modelle und Techniken wird auf die nachfolgenden Beiträge dieses Buches sowie auf die dort angegebenen Quellen verwiesen.

\subsection{Methoden- und Modellbegriff}

Der Methodenbegriff des Business Engineerings entstammt dem Method Engineering [Heym 1993; Gutzwiller 1994; vgl. auch Beitrag von Österle in diesem Band]: Durch eine Methode wird ein Vorgehen in Form von Aktivitäten festgelegt. Die Spezifikation des Ablaufs dieser Aktivitäten hinsichtlich ihrer Reihenfolge und zulässiger Überlappungen wird als Vorgehensmodell bezeichnet. Aktivitäten erzeugen und / oder verwenden ein oder mehrere Ergebnisse. Die Aktivitäten werden dabei aus Sicht des Aufgabenträgers in Form von Rollen zusammengefasst. Die jeweilige Vorschrift zur Erstellung (und damit zur Dokumentation) der Ergebnisse wird als Technik bezeichnet. Werkzeuge können benutzt werden, um die Anwendung einer Technik zu unterstützen. Die in den verschiedenen Ergebnissen repräsentierten Informationen und deren Zusammenhänge können im Informationsmodell der Methode abgebildet werden. Eine Methode lässt sich somit durch ihr Aktivitätsmodell (d.h. die Menge aller Aktivitätsspezifikationen), das Vorgehensmodell, das Ergebnismodell (d.h. die Menge aller Ergebnisspezifikationen), das Rollenmodell, die zu benutzenden Techniken sowie das Informationsmodell beschreiben. 
Als Modellierung wird der Vorgang der Konstruktion eines Abbilds realer oder gedachter Sachverhalte verstanden, welcher auf der Grundlage der Wahrnehmung dieser Sachverhalte durch den/die Modellierer/in erfolgt und durch den jeweiligen Modellierungszweck beeinflusst wird. Die Vorschrift zur Modellkonstruktion wird als (Konstruktions-)Technik bezeichnet. Von der Technik als Vorschrift zur Erstellung eines Modells ist die Sprache oder Notation zu unterscheiden, mit der das Modell beschrieben wird. Allerdings können bestimmte Sprachen/Notationen an bestimmte Techniken gebunden sein und umgekehrt. Modelle entstehen als Ergebnisse der Modellierung, d.h. als durch bestimmte Wahrnehmung und bestimmten Zweck beeinflusste Abbilder realer oder gedachter Sachverhalte [vgl. Schütte 1998]. Modelle können deshalb auch niemals als pauschal richtig oder falsch, sondern lediglich als in einem bestimmten Kontext gültig oder konsistent bezeichnet werden.

Modellierung dient unterschiedlichen Zwecken: [vgl. Leist 2002]

- Schulungszweck: Vermittlung organisatorischer Abläufe oder der Funktionalitäten eines Informationssystems an dessen Nutzer

- Kommunikationsbasis: Unterstützung der Diskussion innerhalb und ausserhalb eines Unternehmens sowie zwischen verschiedenen, an einem Entwicklungsprojekt beteiligten Rollen über organisatorische Abläufe, Funktionalitäten eines Informationssystems, Informationsobjekte usw.

- Analysezweck: Schaffung einer Grundlage für Schwachstellenanalysen, Optimierung oder Controlling organisatorischer Abläufe

- Gestaltungs- und Entwicklungszweck: Schaffung einer konzeptionellen Grundlage für die Eigenentwicklung von Applikationen bzw. die Integration von Standardsoftware oder auch für die Gestaltung neuer bzw. Anpassung bestehender organisatorischer Abläufe

Zur Verdeutlichung der Methoden- und Modellbegriffe des Business Engineerings mag das Beispiel „Transformationsmethode“ dienen, das in [Österle/Blessing 2000] skizziert wird: Die Planung und Umsetzung neuer Geschäftslösungen (,Transformation“) wird beschrieben als Netz verschiedener Aktivitäten wie z.B. Kundenprozessvision spezifizieren, Geschäftslogik spezifizieren, Vertriebskanäle festlegen, Prozesslandkarte erzeugen, Leistungsverzeichnis erzeugen, Aufgabenketten spezifizieren, Anwendungsstruktur ableiten, Stakeholder-Map spezifizieren, Projektaufträge definieren etc.. Diese Aktivitäten laufen entsprechend der Festlegungen der Methode teilweise parallel, teilweise nacheinander ab (Vorgehensmodell). So wie im Verlaufe der „Erzeugung der Leistungsverzeichnisses“ (Aktivität) aufgrund bestimmter Konstruktionsvorschriften (Technik) ein Dokument des Typs „Leistungsverzeichnis“ (Ergebnis) erzeugt wird, erzeugen auch alle anderen Aktivitäten bei Verwendung der jeweils empfohlenen Technik Ergebnisdokumente eines bestimmten Typs. Die entstehenden Ergebnisdokumente sind teilweise strukturierte Dokumente (z.B. Vertriebskanalauswahl, Leistungsverzeichnis, Projektauftrag), teilweise Graphen (z.B. Prozesslandkarte, Aufgabenkette). Sowohl Dokumente wie auch Graphen sind Modelle, da sie als Abbild realer oder gedachter Sachverhalte zu einem bestimmten Zweck konstruiert wurden. 
Im Zentrum der Betrachtungen dieses Beitrags stehen Ergebnistypen bzw. Modelltypen als Ergebnis bestimmter (Konstruktions-)Techniken. Für die detaillierte Beschreibung der Techniken, Aktivitäten, Rollen etc. wird auf spezifische Beiträge in diesem Buch sowie in den referenzierten Publikationen verwiesen.

\subsection{Modelltypen}

Zwar lässt die Vielzahl möglicher Wahrnehmungen und Modellierungszwecke keine exakte Typisierung von Modellen zu. Die folgenden Dimensionen können jedoch zu einer Strukturierung herangezogen werden:

- Ist vs. Soll: Ist-Modelle bilden bestehende Sachverhalte ab. Soll- oder Referenzmodelle bilden dagegen erwünschte oder empfohlene Sachverhalte ab [vgl. Schütte 1998, S. 59].

- Generalisierung vs. Spezialisierung: Spezielle Modelle bilden Sachverhalte in einem bestimmten Anwendungskontext ab. Generalisierte (oder generische [vgl. Leist 2002, S. 6]) Modelle bilden Sachverhalte unter mehr oder weniger starker Ausblendung des Anwendungskontextes ab. Während ein spezielles Modell nur einen oder wenige bestimmte Anwendungskontexte abbildet, sind generische Modelle für eine Vielzahl von Anwendungskontexten gültig.

- Aggregation vs. Dekomposition: Architekturmodelle („Modelle der Gesamtheit" [S. Zanetti, personal communication]) bilden Zusammenhänge zwischen Komponenten auf hohem Abstraktionsniveau ab. Detailmodelle (,Modelle des Einzelnen" [S. Zanetti, personal communication]) beschränken sich auf die Abbildung einer bestimmten Komponente des Architekturmodells, z.B. hinsichtlich ihrer Strukturierung und / oder ihres Verhaltens. Sichtenmodelle beschränken sich auf die Abbildung eines bestimmten Teilaspekts einer Komponente eines abstrakteren Modells. Auf jeder Stufe (Architekturmodell, Detailmodell, Sichtenmodell) sind Unterstufen denkbar, die sich z.B. durch die Granularität der Systemstrukturierung oder die Glättung des Systemverhaltens unterscheiden. Gerade wenn Zwischenstufen existieren, verschwimmt jedoch die Grenze zwischen detaillierten, d.h. „feinen“ Architekturmodellen und aggregierten, d.h. „groben“ Detailmodellen. Die Unterscheidung wird im Folgenden deshalb nur dann benutzt, wenn Modellierungstechniken einen bestimmten Aggregationsgrad bzw. -bereich implizieren.

In Abbildung 1 werden der Generalisierungs-Spezialisierungs-Zusammenhang und der Aggregations-Dekompositions-Zusammenhang in Anlehnung an [Born 2002] am Beispiel des Prozesses „Memo erstellen“ illustriert. In der Dimension „Generalisierung-Spezialisierung“" wird der Prozess „Memo erstellen“ durch den Prozess „Dokument erstellen“ generalisiert und durch die Prozesse „Besprechungseinladung erstellen“ und „Protokoll erstellen“ spezialisiert. In der Dimension „Aggregation-Dekomposition“ wird der Prozess „Memo erstellen“ in den Prozess „Kontakte pflegen“ aggregiert und in die Prozesse „Adressat definieren“, „Text erstellen“, „Memo versenden“ und „Memo ablegen“ dekomponiert. Generalisierung / Spezialisierung auf der einen und Aggregation / Dekomposition auf der 
anderen Seite eignen sich sehr gut zur „Navigation“ in komplexen Modellsystemen [vgl. Malone 1998].

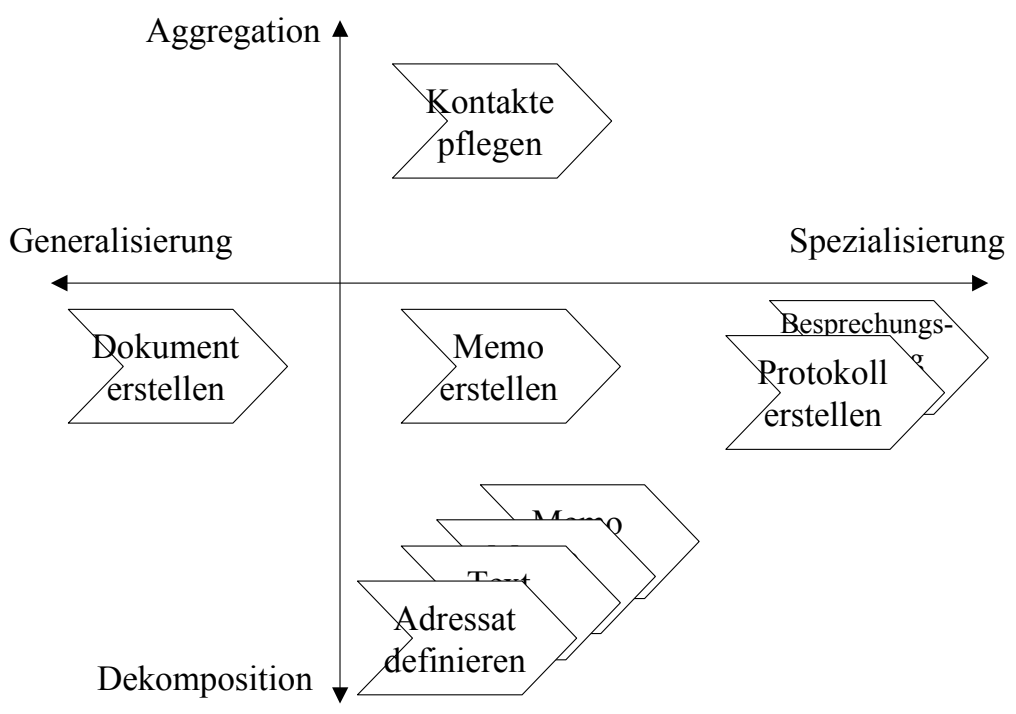

Abbildung 1: Zusammenhänge zwischen (Prozess-)Modellen

Im Hinblick auf syntaktische und semantische Präzisierung werden informelle, semiformale und formale Modelle unterschieden. Informelle Modelle umfassen keine vollständige und / oder eindeutige Beschreibungssyntax (z.B. natürliche Sprache, Listen). Semiformale Modelle umfassen zwar eine präzise Syntax, konkrete Konstruktionsregeln und begrenzte Qualitätsprüfungen (z.B. Entity Relationship-Modell) können aber nicht auf der Grundlage einer formalen Theorie verifiziert werden. Nur für formale Modelle (z.B. Petri-Netze, Modelle auf der Grundlage der Prädikatenlogik) ist neben der Syntax auch die Semantik ausreichend präzisiert, um z.B. ihre Vollständigkeit nachweisen zu können [vgl. Fraser et al. 1994, S. 79].

Statische Modelle bilden einen bestimmten Zustand ab (z.B. Prozesslandkarte). Komparativ statische Modelle bilden mehrere Zustände ab bzw. erlauben einen Vergleich verschiedener Zustände (z.B. Reifegradmodell). Dynamische Modelle bilden einen Ablauf ab (z.B. Ablaufkette).

\section{Modellierungsebenen des Business Engineering}

Komplexe Systeme lassen sich aus den unterschiedlichsten Sichten und für die unterschiedlichsten Zwecke modellieren. Zur Bewältigung der Komplexität hat sich die Bildung einer Hierarchie von Modellierungsebenen bewährt. Die Modellie- 
rungsebenen unterscheiden sich dabei hinsichtlich des Aggregationsgrads und / oder der jeweiligen Ziele der Modellbildung. Modelle auf verschiedenen Modellierungsebenen sind hierarchisch miteinander verknüpft, um die Konsistenz der Gesamtabbildung sicher zu stellen.

Einer kurzen Darstellung einiger Ansätze aus der Literatur (Abschnitt 2.1 [vgl. Leist 2002, S. 11-17]) folgt die Einführung der im Business Engineering verwendeten Modellierungsebenenhierarchie (Abschnitt 2.2).

\subsection{Verschiedene Ansätze zur Ebenenbildung}

Die „multiperspektivische Unternehmensmodellierung“ unterscheidet drei Ebenen: [vgl. Frank 1994, S. 167-170]

- Strategische Perspektive: Diese Sicht enthält die Formulierung der Ziele des Unternehmens sowie den Entwurf und die Bewertung langfristiger Strategien.

- Organisatorische Perspektive: Diese Sicht enthält die Gestaltung und Durchführung arbeitsteiliger Handlungen im Unternehmen.

- Informationssystem-Perspektive: Diese Sicht enthält den Entwurf, die Implementierung und den Betrieb von Informationssystemen.

Das Business Engineering stellt den Veränderungsaspekt in den Mittelpunkt. Es werden drei ähnliche Ebenen unterschieden: [vgl. Österle/Blessing 2000, S. 77]

- Strategie: Diese Sicht beschreibt das Marktumfeld, die strategischen Geschäftsfelder sowie die Marktleistungen des Unternehmens.

- Prozess: Diese Sicht definiert die Prozessleistungen, die arbeitsteiligen Abläufe, die Organisationsstruktur und die Informationsstruktur.

- Informationssystem: Diese Sicht umfasst die Applikationen, einschliesslich der unterstützten Funktionen, IT-Komponenten und Datenstrukturen sowie deren Beziehungen untereinander.

Das ,semantische Objektmodell“ (SOM) spezifiziert zusätzlich zu ModellierungsArchitekturebenen auch Vorgehensmodelle. Die Architekturebenen sind dabei mit den beiden vorgenannten Ansätzen vergleichbar: [vgl. Ferstl/Sinz 1995, S. 212]

- Unternehmensplan: Auf Grundlage der Analyse exogener Erfolgsfaktoren (Chancen und Risiken) und endogener Erfolgsfaktoren (Stärken und Schwächen) werden Unternehmens-, Markt- und Funktionalstrategien festgelegt, Wertschöpfungsketten definiert und Ziele spezifiziert.

- Geschäftsprozessmodelle: Der Unternehmensplan wird durch Geschäftsprozessmodelle umgesetzt, die durch Leistungsbeziehungen miteinander verbunden sind. Jeder Geschäftsprozess trägt zur Sachzielerfüllung bei und unterstützt Formalziele, Erfolgsfaktoren und Strategien.

- Applikationsmodelle: Geschäftsprozesse können durch Personal und Anlagen, insbesondere aber durch Informationssysteme unterstützt werden. Die dritte Ebene dient der Modellierung von Applikationen und ihrer Integration.

Während sich die Modellierungsebenen dieser drei Ansätze im Wesentlichen durch wachsende Implementierungsnähe auszeichnen, verfolgt die „Architektur 
integrierter Informationssysteme“ (ARIS) den Ansatz, Informationssysteme und Geschäftsprozesse integrativ zu betrachten und diesen Komplex in fünf Sichten zu zerlegen, die direkt auf die nachfolgenden Phasen der Systementwicklung hinführen [vgl. Scheer 1998, S. 36]:

- Funktionssicht: Diese Sicht enthält Vorgänge, die Input-Leistungen zu Output-Leistungen transformieren. Hierzu gehören ebenfalls InformationssystemKomponenten, die solche Vorgänge automatisieren.

- Organisationssicht: Diese Sicht bildet die Aufbauorganisation ab. Dabei werden menschliche oder maschinelle Aufgabenträger unterschieden.

- Datensicht: Diese Sicht enthält die Umfelddaten der Vorgangsbearbeitung (z.B. Lieferanten, Kunden, Produkte, Arbeitspläne, etc.) sowie die Nachrichten, welche Funktionen auslösen bzw. von Funktionen erzeugt werden.

- Leistungssicht: Diese Sicht umfasst alle materiellen und immateriellen Inputund Output-Leistungen einschliesslich der Geldflüsse.

- Steuerungssicht: Während in allen anderen Sichten nur die Beziehungen zwischen den Elementen einer einzelnen Sicht abgebildet werden, erfasst diese Sicht die Beziehungen zwischen den Sichten.

Vergleicht man die skizzierten Ansätze, fällt auf, dass auch in ARIS die Steuerungssicht als übergeordnete „Prozessebene“ betrachtet werden kann, welche Geschäftsprozesse integrativ abbildet; Die anderen ARIS-Sichten beschreiben dann auf dieser Grundlage die jeweils spezifischen Prozessaspekte wie Daten, Funktionen etc.

\subsection{Modellierungsebenen im Business Engineering}

Im Business Engineering wird eine Strategie-, eine Geschäftsprozess- und eine Systemebene unterschieden. Diese Unterscheidung findet sich in allen skizzierten Ansätzen; Lediglich in ARIS fehlt eine explizite Strategieebene. Die drei Modellierungsebenen des Business Engineering repräsentieren eine Zielhierarchie: Zunächst wird die strategische Positionierung einer Unternehmung bzw. Geschäftseinheit spezifiziert (,WAS“). Auf dieser Grundlage wird in einem zweiten Schritt die Organisation spezifiziert („WIE“). Auf dieser Grundlage wird in einem dritten und letzten Schritt die Unterstützung geeigneter Aktivitäten durch Informationssysteme spezifiziert (,WOMIT“).

Die Modellierungsebenen des Business Engineerings lassen sich wie folgt charakterisieren:

- Strategieebene: Auf dieser Ebene wird die Rolle des Unternehmens im Wertschöpfungsnetzwerk festgelegt. Jedes Geschäftsmodell wird dann z.B. durch Festlegung der relevanten Kundenprozesse und -segmente, der Kernkompetenzen bzw. Produktionsprozesse, der Partner, des Brandings usw. spezifiziert. Auf dieser Ebene werden auch die wesentlichen Leistungen des jeweiligen Unternehmens aus der Analyse der Kundenprozesse abgeleitet. 
Die Gestaltungsziele auf dieser Ebene sind die optimale Positionierung im Wertschöpfungsnetzwerk und die optimale Grobstrukturierung des Wertschöpfungsbeitrags des jeweils betrachteten Unternehmens.

- Prozessebene: Auf dieser Ebene werden die zur Umsetzung der Strategien notwendigen Geschäftsprozesse und ihr Zusammenwirken beschrieben; Für jeden Geschäftsprozess werden dann die zu erbringenden Prozessleistungen spezifiziert, die zu deren Erbringung notwendigen Aktivitäten einschl. deren Abfolgen festgelegt, Verantwortlichkeiten definiert. Ausserdem werden auf dieser Ebene Informationsobjekte und -flüsse spezifiziert.

Das Gestaltungsziel auf dieser Ebene ist die optimale Organisation und Führung der Wertschöpfung, wobei zunächst nicht festgelegt wird, welche Aktivitäten in welcher Form durch Applikationen unterstützt werden.

- Systemebene: Auf dieser Ebene wird beschrieben, welche Teilprozesse bzw. Aktivitäten mit Applikationen wie unterstützt werden sollen. Zunächst ist die Applikationsarchitektur festzulegen, die auf aggregierter Ebene Applikationen und ihr Zusammenwirken beschreibt. Für jede Applikationskomponente wird die Modellierung dann soweit notwendig detailliert, wobei recht schnell ein so hoher Komplexitätsgrad erreicht wird, dass die Abbildung nur für eine bestimmte Sicht (z.B. Funktionssicht, Organisationssicht, Datensicht, Leistungssicht) erfolgen kann.

Die Gestaltungsziele auf dieser Ebene sind der optimale Schnitt der Applikationen und im weiteren Verlauf die optimale Wiederverwendung von Implementierungskomponenten (z.B. Modulen und Datenstrukturen bzw. Objekten).

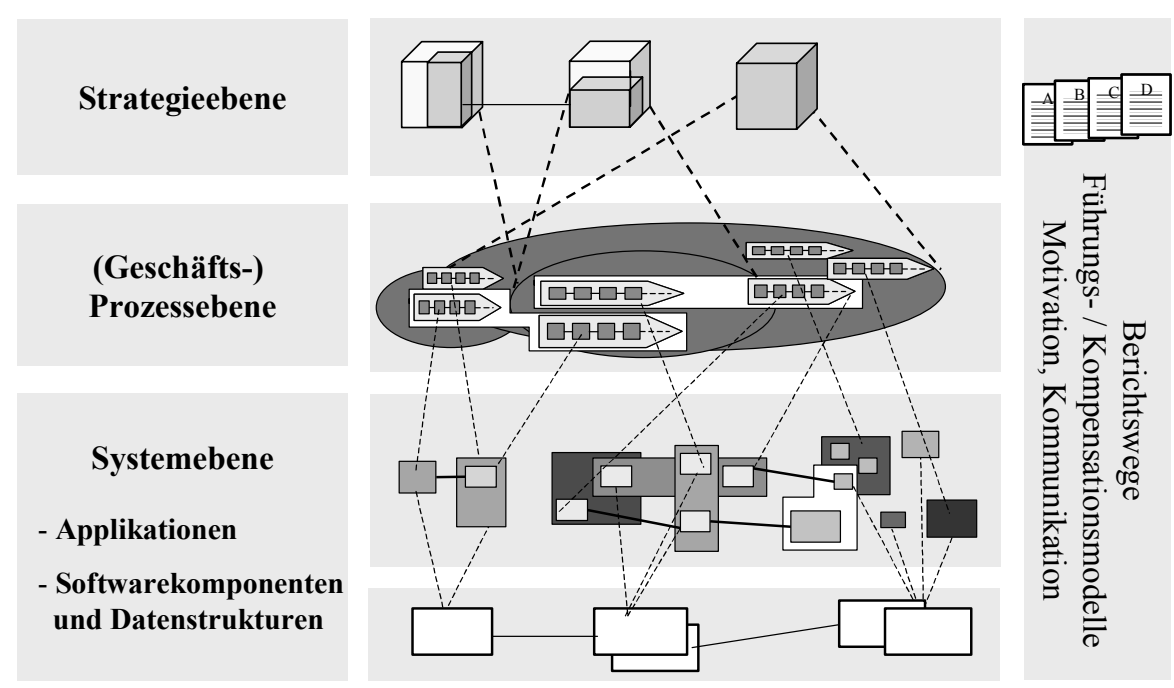

Abbildung 2: Modellierungsebenen des Business Engineering

[in Anlehnung an Leist/Winter 2000]

Abbildung 2 illustriert die Modellierungsebenen und ihre hierarchische Verknüpfung. Auf Strategieebene symbolisieren die Würfel Geschäftsmodellbeschreibungen. Jedem Geschäftsmodell werden auf Prozessebene ein oder mehrere 
Geschäftsprozess(e) zugeordnet, welche die entsprechenden Leistungen erzeugen. Jedem Geschäftsprozess werden auf Systemebene eine oder mehrere Applikation(en) zugeordnet, um geeignete Prozessschritte bzw. Aktivitäten zu unterstützen. Bei der Implementierung der Applikationen wird versucht, möglichst viele Implementierungskomponenten wiederzuverwenden bzw. möglichst grosse Anteile von Standardsoftware zu verwenden.

Parallel zum Durchlaufen der drei Modellierungsebenen sind Querbezüge zu (Unternehmens-)Politik, (Unternehmens- bzw. Veränderungs-)Kultur und Verhalten zu beachten. Traditionell entziehen sich diese „,weichen“ Aspekte von Veränderungsprozessen einer Erfassung durch semiformale Modelle und „harte“ Techniken; In neuester Zeit versuchen verschiedenste Arbeiten (Beispiele finden sich u.a. in diesem Band), diese Lücke zu überwinden. Zur Abbildung der ,sozialen Dynamik" können individuelle Modelle (z.B. Menschenbilder, Motivationstheorien), Gruppenmodelle (z.B. Führungstheorien), Organisationsmodelle (z.B. Projekt- und Innovationsmodelle) bis hin $\mathrm{zu}$ Gesellschaftsmodellen herangezogen werden [S. Zanetti, personal communication].

Die Strategie- und die Prozessebene dienen der rein fachlichen Beschreibung von Organisationszielen, Geschäftsmodellen, Organisationsstruktur, Informationsstruktur, Abläufen und Führung. Auf der Systemebene werden die zur Unterstützung der Geschäftsprozesse dienenden Applikationen zunächst abstrakt und in ihrem Gesamtzusammenhang beschrieben (Applikationsarchitektur). Dieses Gesamtmodell kann dann später, soweit dies zur Unterstützung der Eigenentwicklung oder der Standardsoftwareeinführung notwendig ist, in Teilmodelle zerlegt werden, die für einzelne Applikationskomponenten auf die Funktionssicht, die Organisationssicht, die Datensicht oder die Leistungssicht fokussieren. Erst ganz am Ende wird mit der Planung wiederverwendbarer Implementierungskomponenten und deren systematischer Wiederverwendung von der fachlichen Beschreibung in eine technische Beschreibung übergegangen.

\section{Modelle und Techniken auf Strategieebene}

Auf der Strategieebene wird die Rolle des Unternehmens im Wertschöpfungsnetzwerk festgelegt; Jedes Geschäftsmodell wird dann z.B. durch Festlegung der relevanten Kundenprozesse und -segmente, der Kernkompetenzen bzw. Produktionsprozesse, der Partner, des Brandings usw. spezifiziert. Auf der Analyse der Kundenprozesse basiert die Ableitung der durch das Unternehmen zu erbringenden Leistungen im Wertschöpfungsnetzwerk.

Die Managementlehre, insbesondere deren Richtung „strategisches Management", hat ein umfangreiches Instrumentarium zur Entwicklung, Beschreibung und Kommunikation von Geschäftsstrategien entwickelt [vgl. z.B. MüllerStewens/Lechner 2001]. Die folgenden Ausführungen beziehen dieses Instrumentarium mit ein, beschränken sich jedoch auf solche Aspekte der Beschreibung von Strategien, die für das spätere Verständnis von Geschäftsprozessen und Applikationen notwendig sind. 
Für die Modellierung der Positionierung eines Unternehmens bzw. eines Geschäftsbereichs im Wertschöpfungsnetzwerk und die Grobstrukturierung des Wertschöpfungsbeitrags kommen insbesondere die folgenden drei Modelle in Betracht:

- Das Geschäftsarchitekturmodell beschreibt das Zusammenwirken von Unternehmen bzw. Geschäftsbereichen in Wertschöpfungsnetzwerken.

- Das Geschäftsmodell beschreibt die relevanten Kundenprozesse und -segmente, die Kernkompetenzen bzw. Produktionsprozesse, die Partner, das Branding usw. und bildet damit die wesentlichen Elemente der Unternehmensbzw. Geschäftsbereichsstrategie in unterschiedlichen Detaillierungsgraden ab.

- Das Kundenprozessmodell stellt die Verbindung zwischen den abzudeckenden Kundenprozessen und der Leistungserbringung des jeweiligen Unternehmens bzw. dem jeweils betrachteten Geschäftsbereich her.

Das Geschäftsarchitekturmodell und das Geschäftsmodell stehen zueinander in einer Aggregationsbeziehung. Alle diese Modelle sind im Normalfall statisch, können aber auch zum Soll-/Ist-Vergleich oder zur Reifegradabbildung genutzt werden. Die Modelle können zwar nicht im engeren Sinne als semiformal bezeichnet werden, da die Standardisierung der Syntax und die Formalisierung der Abbildungsregeln noch ausstehen. Die Modelle eröffnen aber einen wesentlich strukturierteren Zugang zur Strategieebene als die traditionell vorherrschende, rein textuelle und damit informelle Dokumentation.

\subsection{Modellierung der Geschäftsarchitektur}

Das Geschäftsarchitekturmodell beschreibt, wie in einem Wertschöpfungsnetzwerk bestimmte Unternehmen bzw. Geschäftseinheiten vom Service Integrator, Shared Service Provider, Exclusive Service Provider und Public Service miteinander über die Business Collaboration Infrastructure zusammenarbeiten, d.h. welche Leistungen ausgetauscht und welche Kundenprozesse abgedeckt werden. Das Geschäftsarchitekturmodell beschreibt nicht die einzelnen Komponenten des Wertschöpfungsnetzwerks, sondern vielmehr deren Zusammenwirken.

Ausgangspunkt der Technik „Geschäftsarchitektur beschreiben“ sind die Kundenprozesse, hinter denen in den meisten Fällen auch spezifische Kundensegmente stehen. Bestimmte Kundenprozesse werden jeweils durch Service Integrators ganzheitlich unterstützt, wobei ein Kundenprozess (bzw. Kundensegment) auch durch verschiedene Service Integrators unterstützt werden kann. Über einen längeren Betrachtungszeitraum hinweg ist dies häufig der Fall, da sich die Unterstützung oft auf Kundenprozesse bezieht, die nur selten oder gar einmalig auftreten (wie z.B. Wohneigentumserwerb): Das Kundensegment „wandert“ dann vom einen Service Integrator zum nächsten.

Service Integrators sowie Service Providers beziehen Leistungskomponenten von Shared Service Providers, Exclusive Service Providers und Public Services. Dabei werden im Normalfall mehrstufige Leistungsketten entstehen. Während Exclusive Service Providers nur einen oder wenige Service Providers bzw. Service Integrators beliefern und deshalb u.U. aus Effizienzgründen direkte Vernetzun- 
gen aufbauen, erfolgen alle anderen Vernetzungen im Normalfall über eine gemeinsame, offene Kollaborationsinfrastruktur. An die Stelle einer Vielzahl bilateraler Vernetzungen zwischen Geschäftsmodellen tritt dann jeweils nur ein einziger „Adapter“ zu dieser gemeinsamen Infrastruktur.

Der direkte Zugriff von Konsumenten auf Leistungskomponenten von Service Providers ist möglich, wenn seitens der Konsumenten die Nutzung eines Service Integrators nicht erwünscht ist oder wenn die zu unterstützenden Prozesse so spezifisch sind, dass eine individuelle Kombination von Leistungskomponenten unausweichlich ist.

Das aggregierte Gesamtmodell des Zusammenwirkens der verschiedenen Rollen wird durch Abbildung 3 illustriert. Aufgrund der besonderen Bedeutung vernetzter Geschäftsarchitekturen für das Business Engineering wird auf dieses Modell in verschiedenen Beiträgen dieses Bandes vertieft eingegangen.

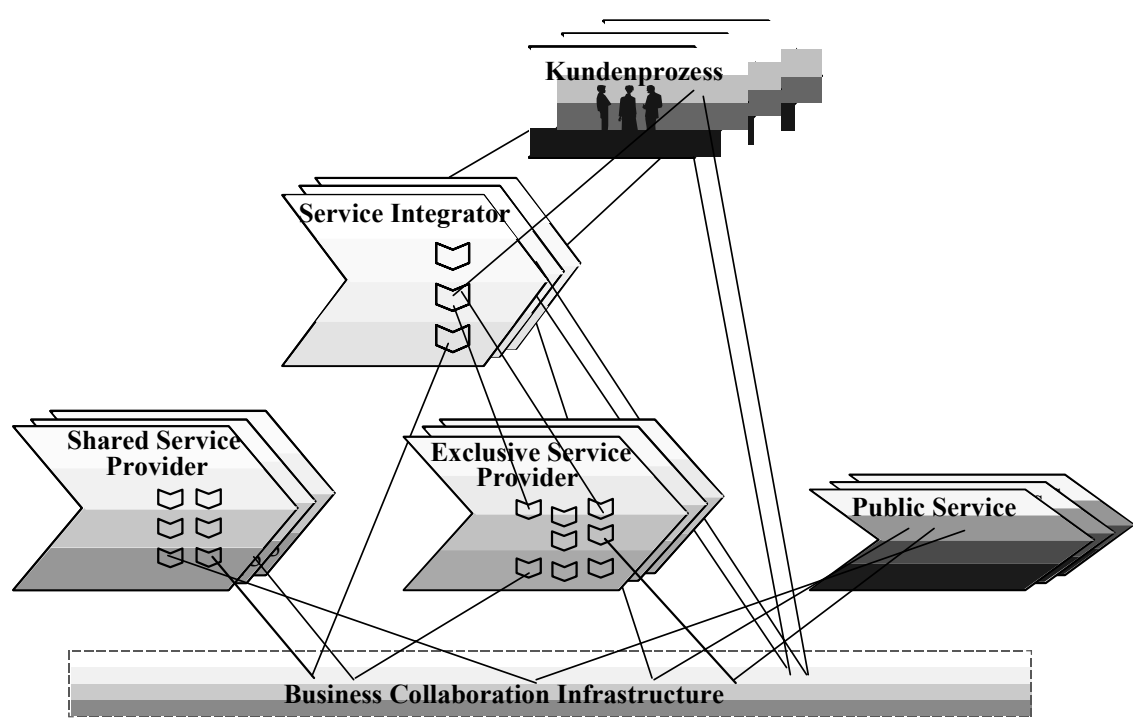

Abbildung 3: (Generisches) Geschäftsarchitekturmodell

[auf Grundlage der Darstellung in Leist/Winter 2000]

Beispiele für eine vernetzte Geschäftsarchitektur finden sich unter anderem bei den genossenschaftlichen Banken oder in der Sparkassenorganisation [vgl. Winter 2002]. Die einzelne genossenschaftliche Bank bzw. Sparkasse „,vor Ort“ besitzt als Service Integrator den direkten Kundenkontakt und das Wissen über die Kundenbeziehungen. Standardisierbare Leistungen wie z.B. Zahlungsverkehr, Depotverwaltung, Handel, Leasing, Versicherungen, Kreditkartenabwicklung oder Bausparen werden durch spezialisierte, zentrale Einheiten (Shared Service Provider) in grossen Stückzahlen erbracht. Spezifische Leistungen wie z.B. Kreditvergabe, Produktentwicklung oder Risikomanagement werden dagegen dezentral oder sogar vor Ort erbracht (Exclusive Service Provider). Als Public Services können in diesem Fall die externe Revision, Kreditsicherungsdienste oder auch der Identitätsnachweis angesehen werden. 


\subsection{Modellierung des Geschäftsmodells}

Das Geschäftsarchitekturmodell beschreibt das Zusammenwirken verschiedener Unternehmen bzw. Geschäftseinheiten. Um das Geschäftsmodell eines bestimmten Unternehmens bzw. einer bestimmten Geschäftseinheit genauer zu beschreiben, wird ein spezifischeres Modell verwendet.

Das Geschäftsmodell beschreibt in bezug auf einen bestimmten Stichtag auf strategischer Ebene den markt-, wertschöpfungs- und potenzialbezogenen Zustand eines Unternehmens oder einer Geschäftseinheit anhand der Ausprägung bestimmter Dimensionen. [vgl. Heinrich 2000, S. 11]

Als Dimensionen, deren Ausprägungen das Geschäftsmodell festlegen, kommen einerseits exogene Faktoren (d.h. äussere, nicht direkt beeinflussbare Rahmenbedingungen) und andererseits endogene Faktoren (d.h. direkt beeinflussbare Aspekte der unternehmerischen Tätigkeit) in Betracht. Im Beitrag von Heinrich/Leist in diesem Band wird auf Grundlage der ausführlichen Darstellung in [Heinrich 2000] die Technik zur Erstellung des Geschäftsmodells zusammengefasst.

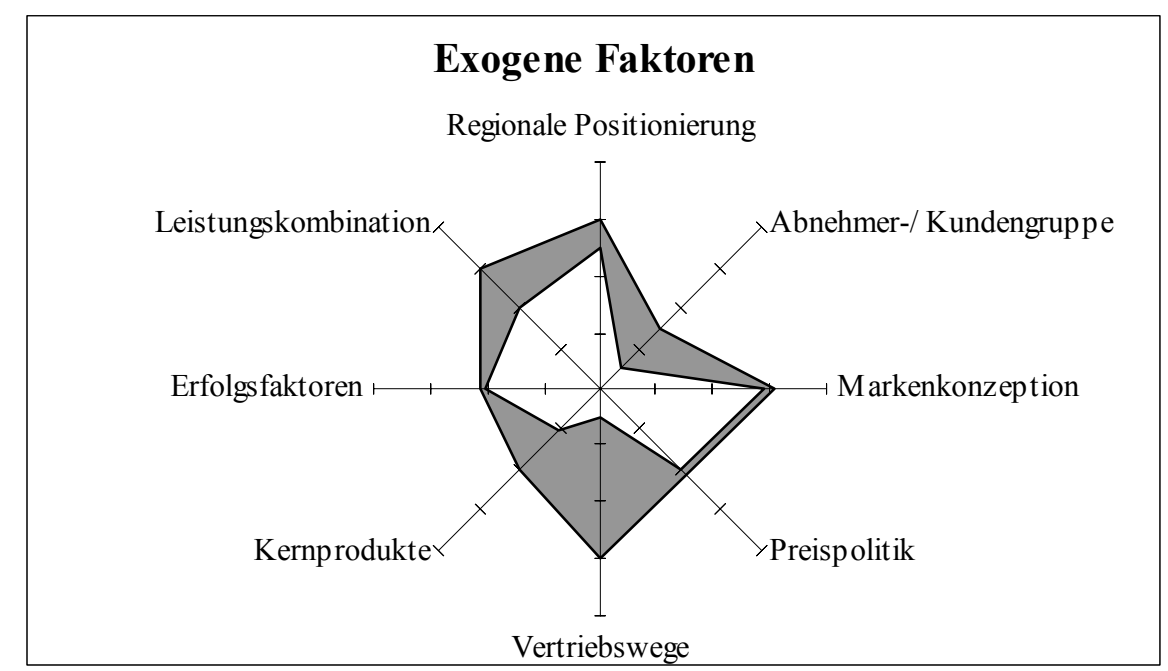

Abbildung 4: Visualisierung der exogenen Faktoren des Geschäftsmodells

Abbildung 4 und Abbildung 5 illustrieren ein bestimmtes Geschäftsmodell, wobei aus Gründen der Übersichtlichkeit auf die Abbildung der verschiedenen Ausprägungen der Dimensionen verzichtet wurde. Mit dieser Technik könnte auch die Veränderung eines Geschäftsmodells abgebildet werden oder es könnten Geschäftsmodelle verschiedener Unternehmen bzw. Geschäftseinheiten verglichen werden. Als Beispiel wird in Anlehnung an [Heinrich 2000, S. 58-63] das deutsche Privatkundengeschäft einer beratungsorientierten Bank betrachtet. Aus der Analyse der in Dokumentenform vorliegenden Geschäftsstrategie sowie aus Ge- 
sprächen mit Geschäftsleitungsmitgliedern ergeben sich bei den exogenen Dimensionen (siehe Abbildung 4) neben trivialen Einordnungen wie z.B. regionale Positionierung „Deutschland“ auch schwierige Festlegungen wie z.B. Kunden-/Abnehmergruppe „materiell, mittleres Potenzial“ oder Markenkonzeption „Convenience“. Das Leistungsangebot ist eher breit. Bei den Vertriebswegen bestehen zwar Prioritäten, die aber nicht fokussiert werden können oder sollen. Bei den endogenen Ausprägungen des betrachteten Geschäftsmodells (siehe Abbildung 5) gestaltet sich die Abbildung klarer, da die Geschäftsmodell-Dimensionen meist in Form von Graden und nicht durch Enumeration von Ausprägungen strukturiert sind. Die Vertriebswege sind weitgehend koordiniert und es wird eine intensive Kooperation mit Partnern im Wertschöpfungsnetzwerk gepflegt. Als Kompetenzquellen werden hauptsächlich Reputation und eigene Mitarbeiter angesehen; Diese Kompetenzen werden synergie- und outputorientiert eingesetzt. Sowohl die Organisation wie auch die Unternehmenskultur können als eher modern bezeichnet werden, d.h. sind dezentral, flach, veränderungs- und individualitätsorientiert. Weitere Beispiele für die Nutzung dieser Technik zur Modellierung des Geschäftsmodells finden sich in [Heinrich 2002a, Braun 2002, Reich/Stucki 2002 und [Flück 2002].

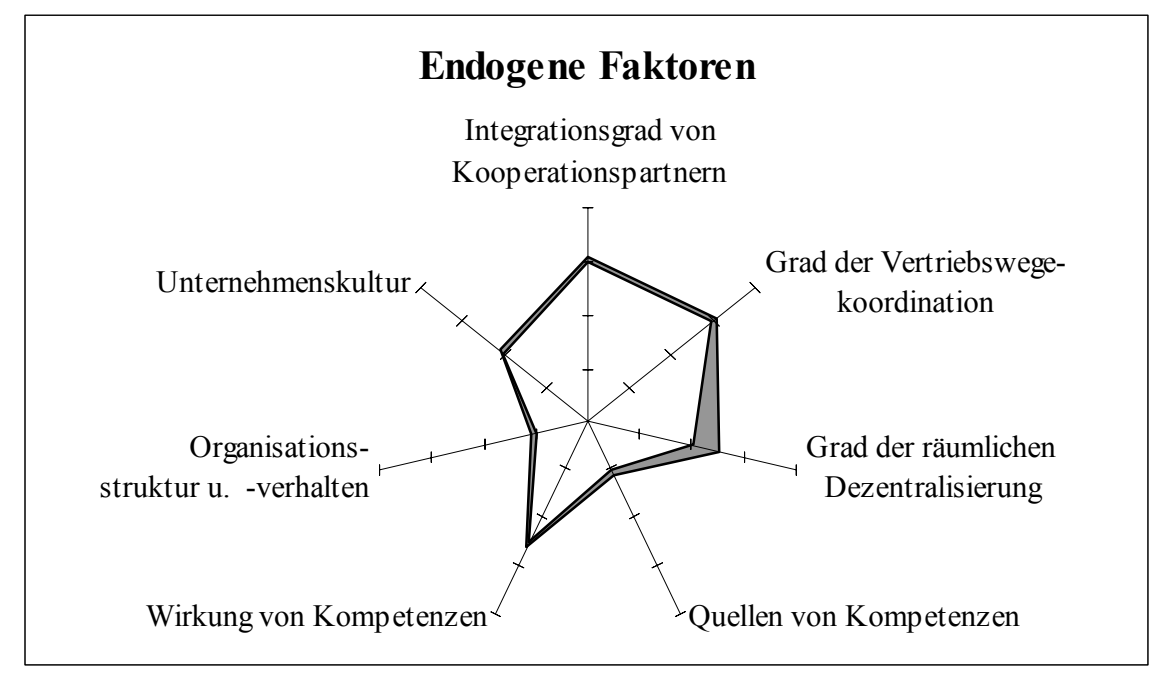

Abbildung 5: Visualisierung der endogenen Faktoren des Geschäftsmodells

Aus dem hier dargestellten multidimensionalen Modell können durch Selektion und Verschmelzung von Dimensionen vereinfachte Modelle gewonnen werden. Je einfacher diese Modelle sind, desto besser lassen sich Längsschnitts- oder Querschnittsvergleiche visualisieren. Ein vierdimensionales Modell mit den Dimensionen „Grad der Einbindung von Kooperationspartnern“, „Dynamik der Organisation“, „Orientierung an Kundenprozessen (vs. Produktionsprozessen)“ und „systematische, integrierte IT-Nutzung" findet sich zur Messung des E-CommerceReifegrads in [Baumöl et al. 2002]. Ein auf dem Vorschlag in [Langlinais/deLeon 1999] basierendes zweidimensionales Modell mit den Dimensionen „Orientierung an Kundenprozessen (vs. Produktionsprozessen)“ und „Virtualisierung von Leis- 
tungszugang und / oder Leistung“ findet sich zur Darstellung des Realisierungsgrads des „Geschäftsmodells des Informationszeitalters“ in [Österle/Winter 2000].

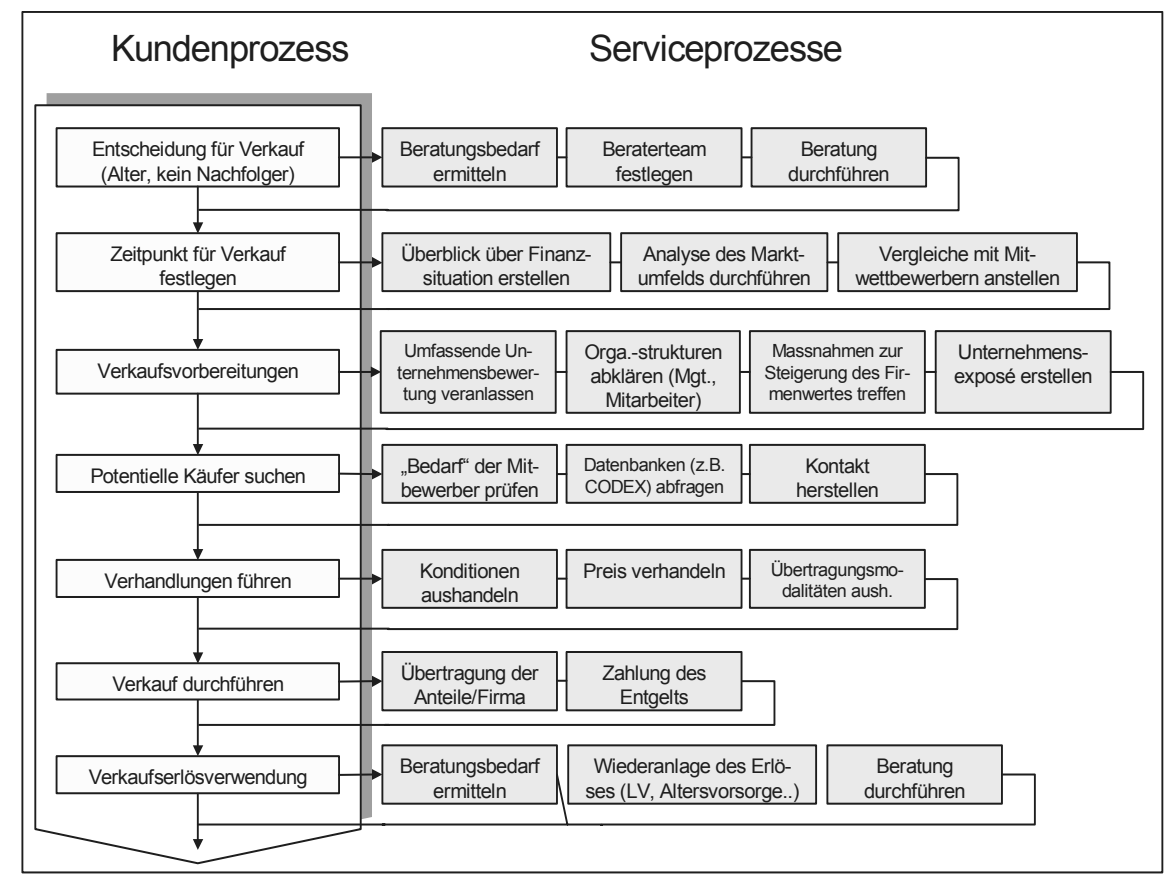

Abbildung 6: Modell zur Ableitung von Leistungen aus der Kundenprozessanalyse [in Anlehnung an Heinrich 2002b, S.87] 


\subsection{Ableitung von Leistungen aus der Analyse von Kundenprozessen}

Das Geschäftsmodell beschreibt die Ausprägungen eines Unternehmens bzw. einer Geschäftseinheit in bezug auf bestimmte vorgefundene (z.B. Markt) und gestaltbare (z.B. Produkte, Organisation) Dimensionen.

Als Grundlage für die nachfolgende Prozessebene müssen jedoch noch die wesentlichen, selbst zu erstellenden Leistungen identifiziert werden. Grundlage dafür ist die Analyse der abzudeckenden Kundenprozesse. Diese Analyse kann beispielsweise mit Hilfe des in [Heinrich 2002b] vorgestellten Modells und der dort beschriebenen Technik zur Festlegung der Vertriebsstrategie durchgeführt werden.

Zunächst werden die verschiedenen Teilaspekte oder Teilschritte des zu unterstützenden Kundenprozesses und deren Abfolge strukturiert. Auf dieser Grundlage wird für jeden Teilaspekt bzw. Teilschritt geprüft, welche Teilleistungen erbracht werden müssen, um den jeweiligen Teilaspekt bzw. Teilschritt zu unterstützen. In nachfolgenden Schritten ist zu prüfen, ob die jeweiligen Teilleistungen auf Grundlage des Geschäftsmodells des jeweiligen Unternehmens bzw. der jeweiligen Geschäftseinheit selbst erbracht werden sollten oder besser fremdbezogen werden. Schliesslich sind geeignete Bündelungen und Vertriebskanäle zu identifizieren, die sich nur auf die eigenerstellten Teilleistungen, aber auch auf zu integrierende Fremdleistungen beziehen.

Abbildung 6 illustriert das Ergebnis der ersten beiden Schritte des hier skizzierten Vorgehens am Beispiel des Kundenprozesses „Unternehmernachfolge“ für Kleinunternehmen aus Sicht des Privatkundengeschäfts einer Bank. Im nächsten Schritt wäre zu entscheiden, welche der auf der rechten Seite identifizierten Teilleistungen selbst und welche durch Partner erbracht werden sollen. In [Fey et al. 2000] wird diese Technik zur Ableitung der Prozessleistungen eines Service Integrators genutzt, der den Kundenprozess „Wohneigentum erwerben“ ganzheitlich unterstützen will.

\section{$4 \quad$ Modelle und Techniken auf Prozessebene}

Während auf der Strategieebene „WAS“-Fragen beantwortet werden, dient die Prozessebene zur Festlegung des „WIE“: Hier werden die Geschäftsprozesse und ihr Zusammenwirken beschrieben. Für jeden Geschäftsprozess werden detailliert die zu erbringenden Prozessleistungen spezifiziert, die dazu notwendigen Aktivitäten einschliesslich deren Abfolgen festgelegt und die Verantwortlichkeiten definiert. Auf Prozessebene ist auch die aggregierte Festlegung der wesentlichen Informationsobjekte und Informationsflüsse anzusiedeln. 
Das Gestaltungsziel der Prozessebene ist die optimale Organisation der Wertschöpfung. „Optimal“ heisst in diesem Zusammenhang, dass die definierten Geschäftsprozesse effektiv geführt werden können und dass effiziente Schnittstellen zwischen Geschäftsprozessen geschaffen werden. Zunächst ist es dabei unerheblich, welche Aktivitäten in welcher Form durch Applikationen unterstützt werden. Neben der Modellierung der Organisation findet auf Prozessebene auch die Abbildung der wichtigsten Informationsstrukturen und -flüsse aus fachlicher Sicht statt. Deshalb kommen für die Prozessebene insbesondere die folgenden drei Modelle in Betracht:

- Das Prozessarchitekturmodell beschreibt das Zusammenwirken der Geschäftsprozesse auf Unternehmens- bzw. Geschäftsbereichsebene.

- Das Prozessmodell beschreibt die Prozessleistungen, die Prozessführung sowie die Abläufe und Verantwortlichkeiten und bildet damit die wesentlichen Elemente von Geschäftsprozessen in unterschiedlichen Detaillierungsgraden ab.

- Das Informationsmodell stellt die benötigten und erzeugten Informationsobjekte in ihrem Gesamtzusammenhang dar. Dabei ist das Informationsmodell der Prozessebene vom Informationsmodell einer Methode zu unterscheiden: Während Methoden-Informationsmodelle die verschiedenen Ergebniskomponenten einer Methode und ihre Verknüpfungen bzw. Abhängigkeiten repräsentieren, stellen Informationsmodelle auf Prozessebene die von den modellierten Prozessen benutzten bzw. erzeugten Informationen und ihre Verknüpfungen bzw. Abhängigkeiten dar.

Das Prozessarchitekturmodell und das Prozessmodell stehen zueinander in einer Aggregationsbeziehung. Während das Prozessmodell dynamisch ist, sind das Prozessarchitekturmodell und das Informationsmodell im Normalfall statisch, obwohl bei letzterem auch dynamische Aspekte (z.B. „Datenflüsse“, dynamische Konsistenz) abgebildet oder zumindest impliziert werden können. Je detaillierter die vorgenommenen Abbildungen sind, desto formalere Modellvarianten können genutzt werden (z.B. Petrinetze für die Ablaufmodellierung). Prozessmodelle können u.U. durch Simulation getestet oder sogar optimiert werden.

\subsection{Modellierung der Prozessarchitektur}

In einem Unternehmen bzw. einer Geschäftseinheit wirken verschiedene Typen von Geschäftsprozessen zusammen: [Österle 1995; Rüegg-Stürm 2000]

- Leistungsprozesse (oder Geschäftsprozesse im engeren Sinne) erzeugen Leistungen für Prozesskunden. Der Kundenbegriff schliesst dabei interne Kunden (z.B. andere als die zu modellierende Geschäftseinheit) ein.

- Unterstützungsprozesse unterstützen die Leistungsprozesse durch Vorleistungen, d.h. durch Leistungen innerhalb des betrachteten Unternehmens bzw. der betrachteten Geschäftseinheit.

- Führungsprozesse koordinieren die Leistungserstellung, d.h. messen die Zielerfüllung von Leistungs- und Unterstützungsprozessen, intervenieren bei Zielabweichungen und entwickeln das gesamte Leistungssystem weiter. 


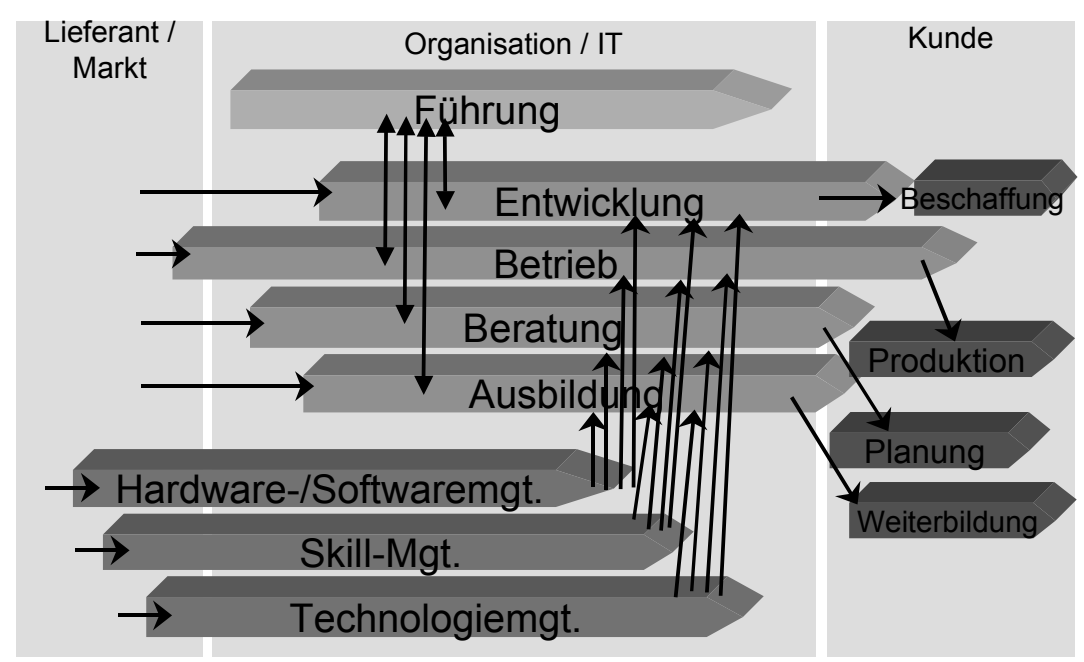

Abbildung 7: Prozessarchitekturmodell

Das Prozessarchitekturmodell (auch: Prozesslandkarte) bildet die wichtigen Geschäftsprozesse und ihre Beziehungen (z.B. Führung, Leistungsverflechtung) ab. Besonders zu beachten ist bereits in dieser frühen Phase der Prozessbeschreibung die Tatsache, dass Geschäftsprozesse im Normalfall mehrere Funktionalbereiche des Unternehmens berühren.

Abbildung 7 zeigt auf Grundlage der Strukturierung aus [Brogli 1996] die Prozesslandkarte eines Informatikunternehmens bzw. Informatikbereichs auf maximalem Aggregationsniveau. Es werden vier Leistungsprozesse (in der Mitte), drei Unterstützungsprozesse (unten), ein Führungsprozess (oben) und vier Kundenprozesse (rechts) sowie deren Beziehungen dargestellt. Aus Gründen der Übersichtlichkeit wurde auf die Benennung der Beziehungen verzichtet.

In [Österle/Blessing 2000] findet sich ein Prozessarchitekturmodell, das zusätzlich auch Beziehungen zu Lieferantenprozessen umfasst. Die Einbeziehung möglichst aller wichtigen „externen“ Beziehungen ist notwendig, um sicher zu stellen, dass auf Prozessebene nicht nur lokale Optimierungsinseln entstehen, sondern das Wertschöpfungsnetzwerk möglichst gesamthaft optimiert wird.

\subsection{Modellierung der Geschäftsprozesse}

Ein Geschäftsprozess ist eine logisch zusammenhängende Kette von Aktivitäten, die in einer vorgegebenen Ablauffolge durchzuführen sind und auf die Erzeugung einer bestimmten Prozessleistung ausgerichtet sind. Ausgelöst durch ein definiertes Ereignis werden bestimmte Einsatzgüter unter Beachtung bestimmter Regeln und durch Einsatz verschiedener Ressourcen zu (Arbeits-)Ergebnissen transformiert.

Basierend auf konzeptionellen Vorarbeiten wie z.B. [Davenport/Short 1989] wurden in der ersten Hälfte der 1990er Jahre verschiedene Techniken zur Ge- 
schäftsprozess-orientierten Organisationsentwicklung vorgeschlagen [vgl. z.B. Österle 1995, Rummler/Brache 1995]. Neben Modellen zur Beschreibung arbeitsteiliger, im Normalfall funktionsübergreifender Abläufe umfassen diese Ansätze meist auch Komponenten zur Identifikation „der richtigen“ Prozesse, geeigneter Führungsgrössen und effizienter Abläufe.

Im Mittelpunkt der Prozessmodellierung steht die Beschreibung der Aktivitätsfolgen (Ablaufplanung). Aus betriebswirtschaftlicher Sicht mindestens ebenso wichtig ist jedoch die Gewährleistung der effektiven Steuerung der Abläufe durch Vorgabe von Leistungs- und Qualitätszielen sowie deren Kontrolle (Prozessführung). Ausgangspunkt der Prozessmodellierung sind die aus der Strategieebene abgeleiteten Festlegungen, welche (Prozess-)Leistungen für welche (Prozess-)Kunden über welche Kanäle abgeboten werden sollen. Diese muss auf Prozessebene verfeinert werden (Leistungsanalyse). Eine vollständige Abdeckung der Prozessebene ist gegeben, wenn neben der Leistungsanalyse, Prozessführung und Ablaufplanung auch die permanente Weiterentwicklung der Geschäftsprozesse geplant und abgebildet wird (Prozessentwicklung) [vgl. Österle 1995].

Bevor Geschäftsprozesse spezifiziert werden können, müssen bestimmte Aspekte ihres Umfelds dokumentiert werden. Soweit bereits bekannt, spielen z.B. die organisatorischen Einheiten und die Standorte eine wichtige Rolle bei der Prozessmodellierung. Soweit möglich, sollten bei der Beschreibung des Umfelds möglichst viele Informationen erfasst werden, die für die späteren Modellierungsund Optimierungsphasen wichtig sind. Für Organisationseinheiten sind dies z.B. die Zahl der Mitarbeiter und die anzusetzenden Kostensätze.

Im Folgenden werden die wichtigsten Techniken und Ergebnisdokumente bzw. Modelle der Prozessmodellierung im Business Engineering skizziert. Ein kurzes Anwendungsbeispiel findet sich im Beitrag „Business Engineering Model“ in diesem Band. Für eine ausführliche Beschreibung der Techniken wird auf [vgl. Österle 1995] verwiesen.

- Ergebnis der Leistungsanalyse ist das Leistungsverzeichnis, ein strukturiertes Dokument, das die Prozessleistungen des jeweils betrachteten Prozesses auf dem jeweils aktuellen Detaillierungsniveau beschreibt.

- Ergebnis der Ablaufplanung ist das Aufgabenkettendiagramm, mit welchem die Abfolge der Aktivitäten und deren Zuordnung zu Verantwortungsbereichen dokumentiert werden. Das Aufgabenkettendiagramm kann darüber hinaus auch Informationen über die Art des Zusammenhangs zwischen Aktivitäten (Sequenz vs. Nebenläufigkeit), auslösende bzw. ausgelöste Ereignisse oder auch erzeugte bzw. benutzte Informationsobjekte enthalten. Ein einfaches Aufgabenkettendiagramm eines Auftragsbearbeitungsprozesses wird in Abbildung 8 dargestellt. In diesem Diagramm bezeichnen graue Rechtecke Aktivitäten, Pfeile sequentielle Abläufe und weisse Rechtecke Organisationseinheiten. Auslösende Ereignisse werden durch Pfeile nach rechts, (Arbeits-)Ergebnisse und ausgelöste Ergebnisse durch normal bzw. fett umrandete Pfeile nach links dargestellt. Die Verantwortlichkeit einer Organisationseinheit für eine Aktivität wird dadurch visualisiert, dass die betreffenden Aktivitäten „über“ die betreffende Organisationseinheit verschoben wird. 
- Zur Detailspezifikation der Aufgaben dient das Aufgabenverzeichnis, das in Form eines strukturierten Dokuments das Mengengerüst, Kapazitätsbedarfe, Qualitätsstandards etc. festhält.

- Im Rahmen der Prozessführung wird festgelegt, welche kritischen Erfolgsfaktoren aus den Organisationszielen abzuleiten sind und durch welche Führungsgrössen sich diese kritischen Erfolgsfaktoren für die entwickelten Prozesse operationalisieren lassen. Die Führungsgrössen werden entsprechenden Aktivitäten mit Sollwerten, Erreichungszeit- und Verantwortlichkeitsangaben zugeordnet.

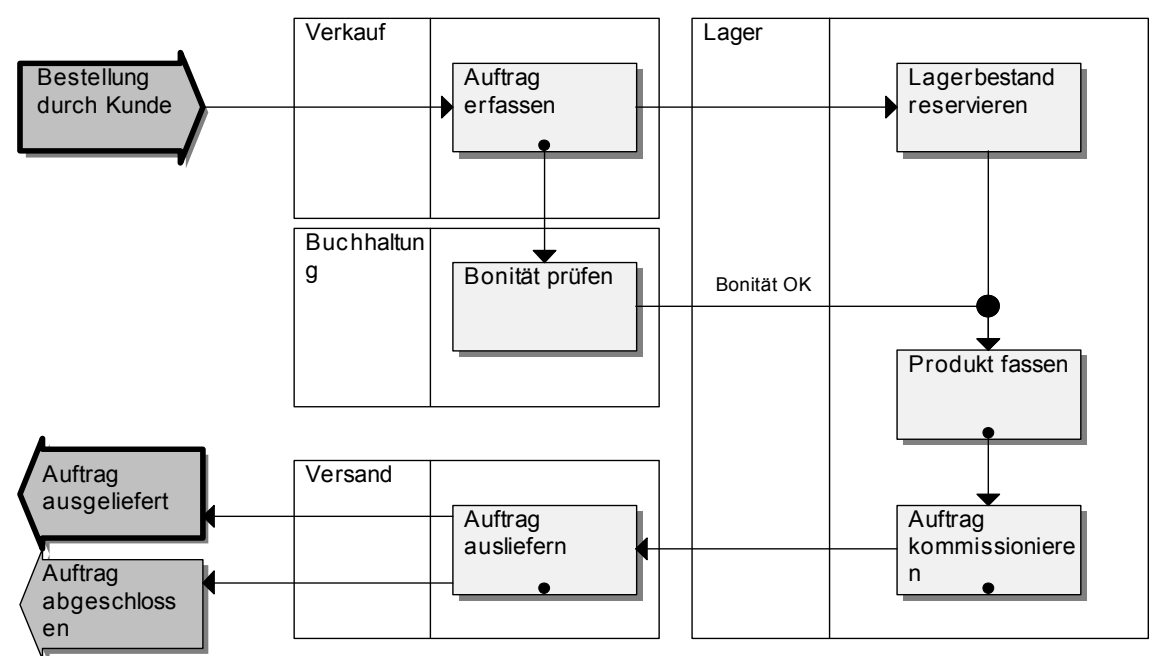

Abbildung 8: Aufgabenkettendiagramm

Bei der Prozessmodellierung werden alle Ergebnisdokumente, vor allem aber Aufgabenkettendiagramme, sukzessiv verfeinert. Die Detaillierung wird durch die Zerlegung von Aktivitäten gesteuert; Organisationseinheiten und Führungsgrössen werden ebenfalls zerlegt, während im Normalfall auf detaillierter Ebene „neue“ Ereignisse, Ergebnisse und Abhängigkeiten zwischen Aktivitäten spezifiziert werden.

Generell können eine „grobe“ Strukturierungsphase und eine „feine“ Strukturierungsphase unterschieden werden.

- Bei der Prozessmodellierung im Groben repräsentieren die Elemente Teilprozesse oder zumindest komplexe Aktivitäten. Als Beziehungstypen werden oft nur Sequenz und Nebenläufigkeit unterschieden.

- Bei der Prozessmodellierung im Detail erfolgt eine Verfeinerung bis hin zur Ebene elementarer Aktivitäten und elementarer Zusammenhänge. Neben den genannten Beziehungstypen können auf detaillierter Ebene Wiederholungen, zusammengesetzte logische Bedingungen o.ä. spezifiziert werden, so dass eine exakte Simulation der Abläufe möglich wird.

Bei jeder Verfeinerung sollte geprüft werden, ob eine feinere Betrachtung wirklich notwendig ist. Das Ziel der Prozessmodellierung, eine möglichst umfassende und 
integrierte Abbildung der Abläufe im Unternehmen, geht nämlich mit zunehmendem Detail mehr und mehr verloren. Andererseits benötigen Softwareentwicklungswerkzeuge oft exakte Spezifikationen, die erst in der Detailmodellierung erfasst werden.

Im Rahmen des ARIS-Ansatzes [vgl. Scheer 1998] wurden in der ersten Hälfte der 1990er Jahre ereignisgesteuerte Prozessketten (EPKs) als Technik zur Prozessmodellierung im Detail entwickelt. Die Benutzung von EPKs unterstützt die Überführung entsprechender Modelle in Softwarelösungen bzw. einen strukturierten Abgleich mit Standardsoftware-Beschreibungen. Für eine ausführliche Beschreibung der EPK-Technik wird auf [Scheer 1998] verwiesen.

\subsection{Modellierung der informationellen Aspekte}

In Prozessmodellen spielen Informationsstrukturen und -flüsse eine untergeordnete Rolle. Zwar ist es möglich, bei der Definition von Aktivitäten Verbindungen zu Informationsobjekten herzustellen; Die Informationsobjekte werden dabei jedoch isoliert und nicht zusammenhängend modelliert. Gerade der Zusammenhang der Informationsobjekte muss jedoch abgebildet werden, um die Konsistenz der erzeugten und verwendeten Informationsobjekte sicher zu stellen.

$\mathrm{Ab}$ Mitte der 70er Jahre wurde in der Folge der Vorstellung des relationalen Datenmodells sowie des Drei-Ebenen-Modells für Datenbanken eine Vielzahl von Modellen vorgeschlagen, die es erlauben, Informationsobjekte sowie deren Beziehungen und Abhängigkeiten aus fachlicher Sicht zu beschreiben. Diese Modelle werden als konzeptionelle (oder semantische) Datenmodelle bezeichnet. Die Gesamtsicht der Informationsstrukturen soll so abgebildet werden, dass

- das Modell von Realisierungsaspekten frei ist und deshalb bei Änderungen der Realisierung nicht geändert werden muss (physische Datenunabhängigkeit) und

- das Modell auch von speziellen Verwendungsaspekten frei ist und deshalb bei Änderungen der Verwendung nicht geändert werden muss (logische Datenunabhängigkeit).

Für die konzeptionelle Datenmodellierung sind viele, stark voneinander abweichende Notationen entwickelt worden; Die vorgeschlagenen Techniken unterscheiden sich darüber hinaus in Vollständigkeit und Mächtigkeit der abbildbaren Realitätsphänomene. Ein Vergleich findet sich z.B. in [Hull/King 1987] und speziell unter dem Gesichtspunkt der semantischen Mächtigkeit der implizierten Konsistenzsicherung in [Winter 1998].

Ergebnis der konzeptionellen Modellierung der Informationsstrukturen sind im Normalfall Diagramme, die Klassen von Informationsobjekten und Zusammenhänge zwischen diesen Klassen (z.B. Generalisierungsbeziehungen vs. Referenzierungen) abbilden. Klassen von Informationsobjekten werden darüber hinaus hinsichtlich ihrer Attribute beschrieben. Bemerkenswert ist, dass die konzeptionelle Modellierung der Informationsstrukturen traditionell nicht-hierarchisch erfolgt, so dass grosse, „flache“ Diagramme entstehen. Einige Techniken sehen zumindest Anordnungsregeln vor, die eine strukturierte Erschliessung des Modells unterstützen (z.B. das SERM-Modell [Sinz 1988]). Das traditionell sukzessiv verfeinernde 
Vorgehen der Prozessmodellierung legt nahe, auch Informationsstrukturen sukzessiv verfeinernd abzubilden. Konsistente Verfeinerungsregeln sind jedoch unbekannt oder nur unter restriktiven Bedingungen anwendbar [vgl. Winter 1996], so dass die Integrität eines aus mehreren Verfeinerungsstufen bestehenden Systems von Informationsmodellen nicht garantiert werden kann.

Konzeptionelle Datenmodelle beschreiben sehr gut den Zusammenhang der abgebildeten Informationsobjekte zu einem bestimmten Zeitpunkt, jedoch nicht (oder zumindest nur sehr eingeschränkt) dynamische Aspekte wie z.B. Datenentstehung, Datenverwendung und Erhaltung der Datenkonsistenz bei Datenänderungen. Deshalb wurden verschiedene Techniken entwickelt, die Prozessmodellierung und Datenmodellierung zusammenführen, z.B. im Rahmen der traditionellen Strukturierten Analyse in Form von Datenflussdiagrammen [Yourdon 1989], durch entsprechend erweiterte Notationen und Techniken zur Informationsmodellierung [vgl. z.B. Winter 1998] oder durch zusätzliche Modellierung von Informationsobjekt-Lebenszyklusdiagrammen [vgl. z.B. Österle 1995].

\section{Modelle und Techniken auf Applikations- und Systemebene}

Während auf der Prozessebene „WIE“-Fragen beantwortet werden, dient die Applikations- und Systemebene zur Festlegung des „WOMIT“: Hier werden die Applikationen und ihr Zusammenwirken beschrieben. Applikationen unterstützen Geschäftsprozesse durch Automatisierung von Aktivitäten und Schnittstellen.

Das Gestaltungsziel der Applikations- und Systemebene ist die optimale Strukturierung und Implementierung der Applikationen. „Optimale Strukturierung“ bedeutet aus fachlicher Sicht, dass die automatisierbaren Aktivitäten effektiv unterstützt werden und dass effiziente Schnittstellen zwischen Applikationen geschaffen werden. „Optimale Implementierung“ bedeutet, dass aus technischer Sicht wiederverwendbare Implementierungskomponenten (Module und Datenstrukturen bzw. Objekte) geschaffen und dann auch tatsächlich wiederverwendet werden.

Aufgrund der Unterschiedlichkeit dieser beiden Ziele können zwei Unterebenen gebildet werden: Auf der Applikationsebene wird aus fachlicher Sicht ausschliesslich das Strukturierungsziel verfolgt, während auf dieser Grundlage auf der Systemebene i.e.S. aus technischer Sicht ausschliesslich das Wiederverwendungsziel verfolgt wird.

Für die Strukturierung der Applikations- und Systemebene werden insbesondere die folgenden drei Modelle bzw. Techniken empfohlen:

- Das Applikationsarchitekturmodell beschreibt das Zusammenwirken der Applikationen innerhalb des betrachteten Unternehmens bzw. Geschäftsbereichs.

- Das Applikationsmodell beschreibt Applikationen aus fachlicher Perspektive in unterschiedlichen Detaillierungsgraden. Die Beschreibung kann sich dabei auf die Datensicht, die Funktionssicht, die Leistungssicht und die Organisationssicht beschränken. 
- Das Komponentenmodell beschreibt wiederverwendbare Komponenten (Module und Datenstrukturen bzw. Objekte), deren Identifikation und deren (Wieder-)Verwendung in Applikationen.

Das Applikationsarchitekturmodell und das Applikationsmodell stehen zueinander in einer Aggregationsbeziehung. Beide Modelle sind generalisierter als das Komponentenmodell, weil in dieses nicht nur fachliche Spezifikationen, sondern auch bestimmte Implementierungsspezifika einfliessen.

\subsection{Modellierung der Applikationsarchitektur}

In Unternehmen wird eine Vielzahl von Applikationen eingesetzt, die sich aus verschiedenen Sichtweisen typisieren lassen. Aus rein technischer Sicht können je nach Installationsort z.B. Applikationen auf Zentralrechnern, Applikationen auf dezentralen Filialsystemen und Applikationen auf persönlichen Rechnern (PCs oder PDAs) unterschieden werden. Die Unterscheidung von Front-Applikationen (z.B. zur Unterstützung der Anlageberatung), Backend-Applikationen (z.B. zur Abwicklung von Produkten) und Basisapplikationen (z.B. Kundenbuchhaltung, Limitenüberwachung) vereinigt technische und fachliche Klassifikationsaspekte. Für eine rein fachliche Typisierung sollten jedoch technische Aspekte wie z.B. der Installationsort oder die Art des „Tiers“ (Interaktion vs. Datenhaltung vs. Funktionalität) keine Rolle spielen. Vielmehr sollte eine an Funktionalitäten, Datenverwendung und Verantwortlichkeiten orientierte Strukturierung erfolgen, die als stabile Grundlage für Entwicklungs-, Integrations- und Rearchitekturprojekte dienen kann.

Ein solches, auf den Methoden „Business Systems Planning“ [vgl. IBM 1983] und „Promet Systems \& Technology Planning“ [vgl. IMG 2000] basierendes Architekturmodell ist erstmals in [Winter 2000] vorgeschlagen worden. Es ordnet Applikationen hinsichtlich ihrer Ausprägungen in Bezug auf Funktionalitäten (z.B. Autorisierung, Zinsberechnung, Saldierung), Informationsobjekten (z.B. Kunde, Risiko; Dimension „Datenverwendung“ in Abbildung 9) und Organisationseinheiten (z.B. Vertrieb KMU, Privatkundenbetreuung UK; Dimension „Verantwortlichkeit" in Abbildung 9) ein. 


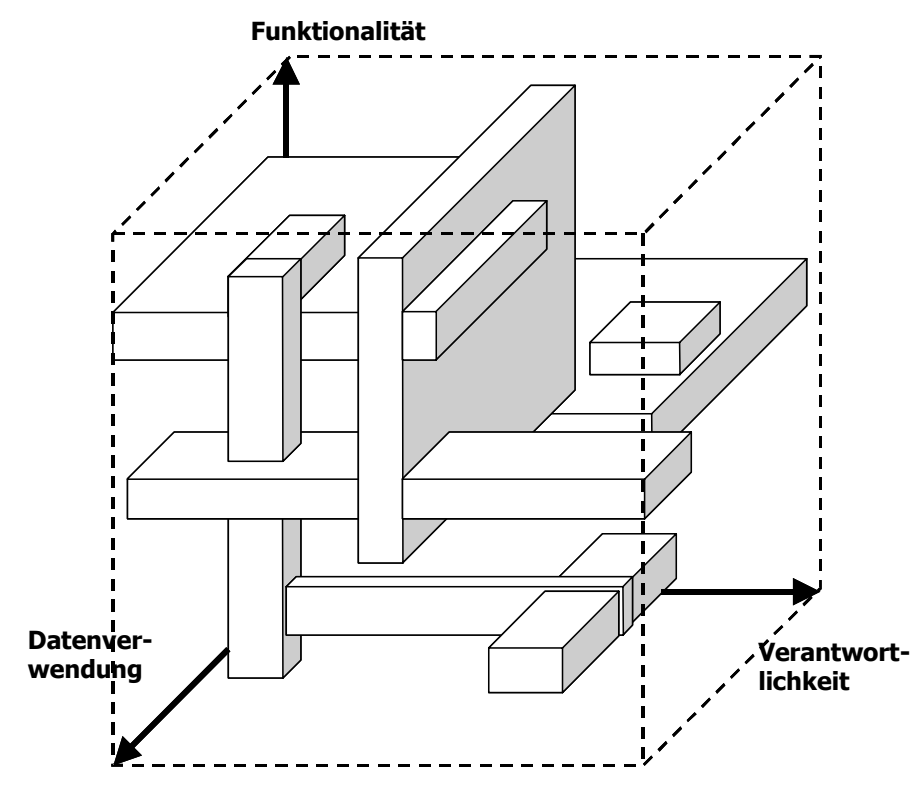

Abbildung 9: Gewachsene Applikationsarchitektur

Abbildung 9 zeigt ein Architekturmodell, das leider für grosse Dienstleistungsunternehmen mit hohem Anteil eigenentwickelter Applikationen typisch ist: Applikationen wurden meist unabhängig voneinander entwickelt und sind entweder um bestimmte Daten herum integriert (z.B. KundenbeziehungsmanagementSysteme), um bestimmte Funktionalitäten herum (z.B. Limitenüberwachung), um bestimmte Produkte bzw. Organisationseinheiten herum (z.B. Hypothekenabwicklung, Zahlungsverkehr) oder verfolgen sogar mehrere dieser Integrationsziele gleichzeitig (z.B. Sicherheitssystem für E-Banking). Es wird deutlich, dass in solchen ,gewachsenen“ Applikationsarchitekturen Redundanzen und Lücken unvermeidlich sind, die durch eine Vielzahl von Integrationsmechanismen wie z.B. eine Vielzahl von Schnittstellenprogrammen, Endverarbeitungen, Enterprise Application Integration o.ä. mühsam kontrolliert bzw. überbrückt werden müssen.

Das Ziel der Architekturplanung auf Applikationsebene muss sein, durch gezielte Entwicklungsprojekte, Einführung geeigneter Standardsoftware-Komponenten und / oder Nutzung moderner Integrationskonzepte (z.B. Data Warehouse, Enterprise Application Integration) diesen Zustand in Richtung einer „sauberen“, Überschneidungen und Lücken vermeidenden Zielarchitektur zu entwickeln. Ein solcher Idealzustand wird in Abbildung 10 dargestellt. 


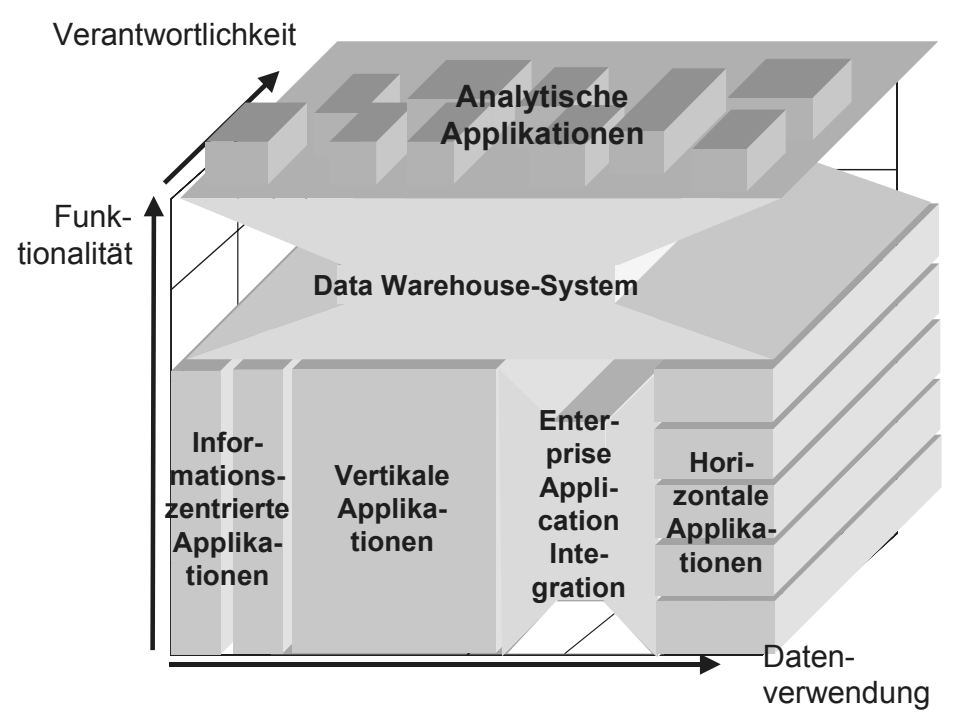

Abbildung 10: Ideal-Applikationsarchitektur

In der idealen Applikationsarchitektur sind Applikationen entweder an einer bestimmten, wiederverwendeten Funktionalität orientiert (Ziel: Wiederverwendung), integrieren Funktionalitäten um bestimmte Informationsobjekte herum (Ziel: Datenintegration) oder integrieren Funktionalitäten um bestimmte Verantwortlichkeiten oder Leistungsprozesse herum (Ziel: Prozessintegration). Bei Applikationen, die um Daten herum integriert werden, sollte unterschieden werden, ob operative Geschäftsprozesse oder ob Führungsprozesse unterstützt werden. Aufgrund der „Mikro-Integration“ durch Applikationsbildung gibt es zwischen Applikationen des gleichen Typs relativ wenige, zwischen Applikationen verschiedener Typen hingegen sehr viele Schnittstellen. Zur Reduktion der Anzahl der Schnittstellen zwischen verschiedenen Applikationstypen wurden verschiedene Typen von „Middleware“ entwickelt. Insgesamt können neben vier Typen von Applikationen (A bis D) drei Typen von Middleware (E bis G) unterschieden werden: [vgl. Winter 2000]

A. Vertikale Applikationen: Diese unterstützen produktspezifische Abwicklungsprozesse, die meist auch mit bestimmten Organisationsbereichen verbunden sind (z.B. Zahlungsverkehr, Hypothekenabwicklung)

B. Horizontale Applikationen: Diese unterstützen produktübergreifende $\mathrm{Zu}$ gangs- und Vertriebsprozesse, die jedoch an einen bestimmten Kanal gebunden sind, d.h. um bestimmte Funktionalitäten herum integriert sind (z.B. Phone-Banking inbound, Telefonvertrieb, E-Banking, Geldautomatenbetrieb)

C. Informationszentrierte Applikationen: Diese unterstützen Prozesse, die um bestimmte Gruppen von Informationsobjekten herum integriert sind (z.B. Kundenbeziehungsmanagement, Produktkonfiguration, Tarifierung)

D. Analytische Applikationen: Diese unterstützen Führungsprozesse durch Bereitstellung von Informationen (z.B. Risikomanagement, Kundensegmentierung). 
E. Data Warehouse System: Diese Integrationskomponente entkoppelt vertikale bzw. horizontale Applikationen von den analytischen Applikationen, in dem Daten aus Geschäftsvorfällen historisiert und integriert werden, um damit umfassende, konsistente Entscheidungsgrundlagen herzustellen.

F. Enterprise Application Integration-Infrastruktur: Diese Integrationskomponente entkoppelt verschiedene operative Applikationen untereinander dadurch, dass nicht mehr Schnittstellen von jedem System zu fast jedem anderen System gebaut werden müssen, sondern statt dessen eine nachrichten- und / oder datenorientierte Austauschplattform betrieben wird.

G. Business Collaboration Infrastructure (in Abbildung 10 nicht enthalten): Diese Integrationskomponente nimmt eine ähnliche Rolle wie Enterprise Application Integration ein, integriert allerdings Applikationen in verschiedenen Unternehmen und muss deshalb verteilt bzw. auf Grundlage offener Kommunikationsstandards und standardisierter Integrationsdienste realisiert werden.

\subsection{Applikationsmodellierung aus fachlicher Sicht}

Für die Dokumentation des Fachkonzepts von Applikationen und die nachfolgende Transformation in das DV-Konzept existieren bewährte Ansätze wie beispielsweise Structured Analysis/Structured Design [Yourdon 1989, Yourdon/Constantine 1979]. Diese Ansätze nutzen Modelle wie z.B. die Funktionshierarchie, Datenflussdiagramme auf verschiedenen Detaillierungsstufen und konzeptionelle Fein-Datenmodelle sowie verschiedene, strukturierte Dokumente bis hin zu Pseudocode zur Spezifikation der Systemfunktionalitäten und der Informationsstruktur.

Structured Analysis / Structured Design ist zwar definitionsgemäss implementierungsunabhängig, wird aber dennoch konzeptionell stark von Dateien (zur Implementierung von Informationsstrukturen) und prozeduralem Code (zur Implementierung von Funktionalitäten) geprägt. Deshalb wurden in den 1980er Jahren Modellierungskonzeptionen speziell für integrierte (d.h. datenbankorientierte) betriebswirtschaftliche Applikationen entwickelt. Hier sind insbesondere die Modelle und Techniken zu erwähnen, die im sog. ARIS-Haus [vgl. Scheer 1998] zusammengeführt wurden.

Alternativ bieten objektorientierte Analyse und objektorientierter Entwurf [vgl. Martin/Odell 1992] eine methodische Unterstützung für die integrierte, schrittweise verfeinernde Spezifikation von Systemstruktur und Systemverhalten, wobei teilweise völlig neue Techniken (z.B. use case-Analyse) und eine im Idealfall durchgängige Modellnotation (verschiedene Diagrammtypen der „Unified Modeling Language" UML) eingesetzt werden.

Spezielle Implementierungsumgebungen, wie z.B. Anwendungsgeneratoren, können besondere Spezifikationsanforderungen mit sich bringen (z.B. ereignisgesteuerte Abläufe mit standardisiertem Kontrollfluss), die bereits bei der Erstellung des Fachkonzepts durch entsprechende Techniken unterstützt werden sollten. 
Alle Techniken und Modelle sind nicht spezifisch für das Business Engineering, so dass für deren Beschreibung auf die einschlägige Informatik- bzw. Wirtschaftsinformatik-Literatur verwiesen wird.

\subsection{Modellierung von Komponenten}

Beim Übergang von der fachlichen Sicht zur technischen Sicht treten neue Ziele, wie optimale Nutzung der Implementierungsumgebung und insbesondere optimale Wiederverwendung, an die Stelle von bisherigen Zielen, wie korrekter Dokumentation der Anforderungen oder konsistenter Abbildung dieser Anforderungen in den verschiedenen Teilmodellen. Beispielsweise werden im Structured Design Modulkandidaten u.a. dadurch identifiziert, dass Funktionsbeschreibungen mit ähnlicher oder identischer Datennutzung unabhängig von der (fachlichen) Einordnung in der Funktionshierarchie zusammengeführt werden [vgl. Yourdon/Constantine 1979]. Traditionell werden Modelle auf Fachkonzeptebene mit Hilfe bestimmter Techniken in automatisierter Form in Initialmodelle der DVbzw. Implementierungsebene transformiert, die dann mit speziellen Techniken überarbeitet und / oder verfeinert werden. So erfolgt z.B. die Ableitung von Modulhierarchien aus Funktionshierarchien und Datennutzungs-Spezifikationen und die Ableitung eines Relationenmodells aus einem konzeptionellen Datenmodell in vielen Modellierungswerkzeugen durch sog. Generatoren in automatisierter Form.

Allerdings passt die Transformierung fachlicher Modelle in implementierungsnahe Modelle nicht zur Zielhierarchie des Business Engineering, da im Normalfall neue, spezifische Lösungen erzeugt werden und bereits existierende Lösungen nicht systematisch wiederverwendet werden. An die Stelle der Transformierung sollte deshalb die Konfigurierung von Implementierungslösungen auf der Grundlage wiederverwendbarer Komponenten (Module und Datenstrukturen bzw. Objekte) treten. Die Konfigurierungstechnik gleicht Anforderungen aus fachlicher Sicht mit Komponenteneigenschaften ab und erzeugt eine Ausgangskonfiguration, welche die Anforderungen möglichst gut erfüllt (,Design by Reuse“). Die endgültige Implementierungslösung wird danach durch Anpassung bestehender Komponenten und allenfalls Erzeugung neuer Komponenten erarbeitet. Bei der Anpassung und Erstellung von Komponenten ist dabei der Wiederverwendbarkeit Rechnung zu tragen (,Design for Reuse“).

Komponenten sind hinsichtlich Schnittstellen, Verhalten, Abstimmung, Qualität, Terminologie, Aufgaben und Vermarktung zu beschreiben [vgl. Turowski 2002, S. 3-4]. Als Beschreibungstechniken werden Sprachstandards wie IDL (OMG Interface Description Language, für Schnittstellen), OCL (OMG Object Constraint Language auf UML-Basis, für Verhalten und mit Erweiterungen für Abstimmung), RDF (Resource Description Framework auf XML-Basis, für Terminologie), semantische Informationsmodelle (für Aufgaben) und strukturierte Dokumente (für Vermarktung) vorgeschlagen [vgl. Turowski 2002, S. 4-24]. Für die Identifikation wiederverwendbarer Funktionalitäten (d.h. Komponentenkandidaten) im Business Engineering aus fachlicher und technischer Sicht wurde in [Choinowski 2002] die PIT-Technik vorgeschlagen. 


\section{Werkzeuge}

Werkzeuge sind computergestützte Systeme zur Unterstützung der Anwendung von (Modellierungs-)Techniken. Die Unterstützung kann in unterschiedlichem Umfang erfolgen:

- Projektwerkzeuge: Diese Werkzeuge unterstützen die Methodenanwendung durch Vorgehensanleitungen, vordefinierte Dokumente („Templates“), Verwaltung von Projektdokumenten und Projektmanagementfunktionalitäten. Beispiele sind ASAP Implementation Assistant und ASAP Q\&A-Datenbank.

- Editoren: Diese Werkzeuge unterstützen die Erstellung von Modellen durch Editierfunktionen, Verwaltung maschinenlesbarer Modelle und allenfalls einfache Syntaxprüfungen. Beispiele sind Office-Produkte für Grafik- und Textverarbeitung und Visio. Durch Business Engineering-spezifische Erweiterungen (z.B. SemTalk für Visio) kann der Nutzen der Werkzeugnutzung erheblich erhöht werden.

- Modellierungswerkzeuge: Diese Werkzeuge unterstützen über die Funktionalitäten von Editoren hinaus die Verwaltung und Wiederverwendung von Modellkomponenten sowie die Sicherung der Konsistenz innerhalb eines Modells und zwischen verschiedenen Modellen. Beispiele sind ARIS Toolset, CASEwise und Oracle Designer sowie eingeschränkt (da nur einen oder wenige Ergebnistyp/en unterstützend) ErWin oder Rational Rose.

Generell ist der Werkzeugnutzung entgegen zu halten, dass die menschliche Kreativität mehr oder weniger stark eingeschränkt wird und dass fortgeschrittene Werkzeuge (z.B. Modellierungswerkzeuge) einen oft erheblichen Lern- und Administrationsaufwand mit sich bringen. Oft werden nur isolierte, evtl. noch dazu veraltete Techniken bzw. Modelle unterstützt, so dass eine ganzheitliche Unterstützung komplexer Business Engineering-Projekte nicht möglich ist. Für Modellierungswerkzeuge rentieren sich die Aufwände für Administration und Schulung in jedem Fall erst nach vielen bzw. grossen Projekten, so dass diese Werkzeuge professionellen Inhouse- oder externen Business Engineers vorbehalten bleiben. Dies gilt nicht für Editoren und Projektwerkzeuge, die selektiv auch in kleinen Projekten erfolgreich eingesetzt werden können.

Modellierungswerkzeuge können die Verfeinerung von Modellen sowie den Übergang zwischen Modellierungsphasen (z.B. Prozessebene zu Systemebene) erheblich vereinfachen. Neben der Unterstützung der Technikanwendung und der Qualitätssicherung verfügen einige Werkzeuge auch über Simulations- und Optimierungsfunktionalitäten. So können z.B. in CASEwise und Oracle Designer nach Erfassung von Häufigkeiten, Verteilungen, Einzel- und Gemeinkosten sowie Bearbeitungs-, Liege- und Transportzeiten in Prozessmodellen Durchlaufzeiten, Prozesskosten und kritische Pfade simuliert werden. Fortgeschrittene Modellierungswerkzeuge erlauben darüber hinaus, die Anzahl paralleler Ausführungen zu definieren sowie minimale und maximale Losgrössen $\mathrm{zu}$ definieren. Mit diesen und weiteren Angaben (z.B. zu Verteilungen und Entwicklungen im Zeitverlauf) 
können dann auch komplexe Abläufe simuliert werden. Durch gezielte Parameterveränderung können auf Grundlage solcher Simulationen Durchlaufzeiten verkürzt, Kapazitätsüberschüsse minimiert und Kosten gesenkt werden.

\section{Schlussbetrachtung}

Der maximale Nutzen des ingenieurmässigen Ansatzes zur Gestaltung der Transformation kann realisiert werden, wenn Modelle und Methoden nicht nur partiell und isoliert, sondern möglichst umfassend und integriert verwendet werden. Diese Betrachtung versucht, ausgewählte Modelle (und damit entsprechende Techniken) in einen Zusammenhang zu bringen. Dabei wird zunächst die Drei-EbenenStruktur aus der Zielhierarchie „WAS“-,WIE“-,,WOMIT“ begründet.

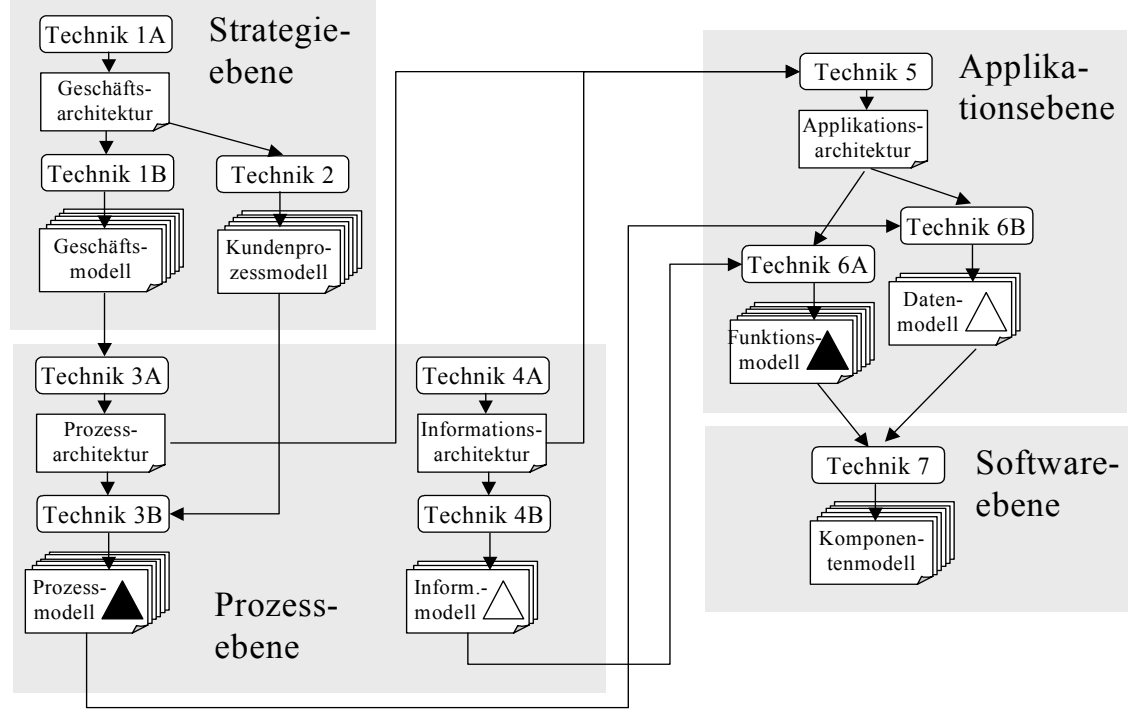

Abbildung 11: Gesamtzusammenhang ausgewählter Modelle und Techniken

Abbildung 11 illustriert den Zusammenhang zwischen den skizzierten Modellen und entsprechenden Konstruktionstechniken. Es fällt auf, dass auf den drei Modellierungsebenen des Business Engineerings (Applikations- und Softwareebene werden als Systemebene gemeinsam betrachtet) in ähnlicher Weise vorgegangen wird, obwohl die jeweiligen Gestaltungsziele völlig unterschiedlich sind: Zunächst wird der jeweilige Gesamtzusammenhang in Architekturmodellen abgebildet (vgl. Geschäfts-, Prozess- und Applikationsarchitektur).

Danach werden die Architekturkomponenten einzeln beschrieben (vgl. Geschäfts-, Prozess- und Applikationsmodell). Die Abbildung kann sukzessiv verfeinernd erfolgen (vgl. insbes. Prozess- und Funktionsmodell, in Abbildung 11 durch eine Pyramide visualisiert) und/oder kann zur Komplexitätsreduktion in verschiedene Sichten zerlegt werden (vgl. Funktions- vs. Datenmodell). 


\section{Literatur}

[Baumöl et al. 2002]

Baumöl, U., Fugmann, T., Stiffel, T., Winter, R., A Concept for the Evaluation of ECommerce-Ability, in: Gangopadhyay, A. (Ed.), Managing Business with Electronic Commerce - Issues and Trends, Idea Group Publishing, Hershey et al., 2002, S. 199-213 [Born 2002]

Born, U., Innovatives und flexibles Prozessmanagement mit dem Phios Process Repository, in: Leist, S., Winter, R., Retail Banking im Informationszeitalter - Integrierte Gestaltung der Geschäfts-, Prozess- und Applikationsebene, Springer, Berlin et al., 2002, S. 149-166

[Braun 2002]

Braun, B., Neues Geschäftsmodell im Hypothekarbereich der CREDIT SUISSE FINANCIAL SERVICES, in: Leist, S., Winter, R., Retail Banking im Informationszeitalter - Integrierte Gestaltung der Geschäfts-, Prozess- und Applikationsebene, Springer, Berlin et al., 2002, S. 93-108

[Brogli 1996]

Brogli, M., Steigerung der Performance von Informatikprozessen - Führungsgrössen, Leistungsmessung und Effizienz im IT-Bereich, Vieweg, Braunschweig/Wiesbaden, 1996

[Choinowski 2002]

Choinowski, S., Das PIT-Modell, in: Leist, S., Winter, R., Retail Banking im Informationszeitalter - Integrierte Gestaltung der Geschäfts-, Prozess- und Applikationsebene, Springer, Berlin et al., 2002, S. 183-206

[Davenport/Short 1989]

Davenport, T.H., Short, J.E., The New Industrial Engineering: Information Technology and Business Process Redesign, Sloan Management Review, 31 (4), 1989, S. 11-27

[Ferstl/Sinz 1995]

Ferstl, O.K., Sinz, E.J., Der Ansatz des Semantischen Objektmodells (SOM) zur Modellierung von Geschäftsprozessen, in: Wirtschaftsinformatik, 37 (3), 1995, S. 209-220

[Fey et al. 2000]

Fey, B., Heibei, G., Müntener, C., Kober, S., Ein Geschäftsmodell für die Finanzindustrie im Informationszeitalter - Das Life Event Management Konzept, in: Österle, H., Winter, R. (Hrsg.), Business Engineering - Auf dem Weg zum Unternehmen des Informationszeitalters, Springer, Berlin et al., 2000, S. 257-270

[Flück 2002]

Flück, B., Identifizierung neuer Prozesse im Finanzdienstleistungsvertrieb, in: Leist, S., Winter, R., Retail Banking im Informationszeitalter - Integrierte Gestaltung der Geschäfts-, Prozess- und Applikationsebene, Springer, Berlin et al., 2002, 167-182

[Frank 1994]

Frank, U., Multiperspektivische Unternehmensmodellierung: theoretischer Hintergrund und Entwurf einer objektorientierten Entwicklungsumgebung, Oldenbourg, München, Wien, 1994 
[Fraser et al. 1994]

Fraser, M.D., Kumar, K., Vaishnavi, V.K., Strategies for Incorporating Formal Specifications in Software Development, in: Communications of the ACM, 37 (10), 1994, S. 74-85

[Gutzwiller 1994]

Gutzwiller, T., Das CC RIM-Referenzmodell für den Entwurf von betrieblichen, transaktionsorientierten Informationssystemen, Physica, Heidelberg, 1994

[Heinrich 2000]

Heinrich, B., Dimensionen zur Beschreibung eines Geschäftsmodells für Kreditinstitute im Bereich Privatkunden, Arbeitsbericht BE HSG/CC BAI/01, Institut für Wirtschaftsinformatik, Universität St. Gallen, St Gallen, Dezember 2000

[Heinrich 2002a]

Heinrich, B., Das Geschäftsmodell als Instrument zur Positionierung des Unternehmens, in: Leist, S.; Winter, R., Retail Banking im Informationszeitalter - Integrierte Gestaltung der Geschäfts-, Prozess- und Applikationsebene, Springer, Berlin et al., 2002, S. 53-71

[Heinrich 2002b]

Heinrich, B., Die konzeptionelle Gestaltung des Multichannel-Vertriebs anhand von Kundenbedürfnissen, in: Leist, S.; Winter, R., Retail Banking im Informationszeitalter Integrierte Gestaltung der Geschäfts-, Prozess- und Applikationsebene, Springer, Berlin et al., 2002, S. 73-92

[Heym 1993]

Heym, M., Methoden-Engineering, Dissertation, Universität St. Gallen, St. Gallen, 1993

[Hull/King 1987]

Hull, R., King, R., Semantic Database Modeling - Survey, Applications, and Research Issues, ACM Computing Surveys, 19 (3), 1987, S. 201-260

[IBM 1983]

IBM Corp. (ed.), Business Systems Planning - Information Systems Planning Guide, Application Manual GE20-0627

[IMG 2000]

IMG AG (Hrsg.), Promet ${ }^{\circledR}$ STP, Methodenhandbuch für die System- und Technologieplanung, Version 1.1, St. Gallen, 2000

[Langlinais/deLeon 1999]

Langlinais, T., deLeon, A., Winning the e-economy with "Intention Value Networks", EIU Strategic Finance, March to May 1999, The Economist Intelligence Unit Ltd., 1999, S. $50-68$

[Leist 2002]

Leist, S., Bankenarchitektur des Informationszeitalters - Zielsetzung und Gestaltungsebenen, in: Leist, S., Winter, R. (Hrsg.), Retail Banking im Informationszeitalter, Springer, Berlin et al., 2002, S. 4-28

[Leist/Winter 2000]

Leist, S., Winter, R., Finanzdienstleistungen im Informationszeitalter - Vision, Referenzmodell und Transformation, in: Belz, C., Bieger, T. (Hrsg.), Dienstleistungskompetenz und innovative Geschäftsmodelle, St. Gallen, Thexis, 2000, S. 150-166

[Malone et al. 1998]

Malone, T.W. et al., Tools for inventing organizations: Toward a handbook of organizational processes, http://ccs.mit.edu/21c/mgtsci/index.htm

[Martin/Odell 1992]

Martin, J., Odell J.J., Object-Oriented Analysis and Design, Prentice-Hall, Englewood Cliffs, 1992 
[Müller-Stewens/Lechner 2001]

Müller-Stewens, G., Lechner, C., Strategisches Management, Schäffer-Poeschel, Stuttgart, 2001

[Österle 1995]

Österle, H., Business Engineering - Prozess- und Systementwicklung, Bd.1: Entwurfstechniken, Berlin et al., Springer, 1995

[Österle/Blessing 2000]

Österle, H., Blessing, D., Business Engineering Model, in: Österle, H., Winter, R. (Hrsg:), Business Engineering - Auf dem Weg zum Unternehmen des Informationszeitalters, Springer, Berlin et al., 2000, S. 61-81

[Österle/Winter 2000]

Österle, H., Winter, R., Business Engineering, in: Österle, H., Winter, R. (Hrsg:), Business Engineering - Auf dem Weg zum Unternehmen des Informationszeitalters, Springer, Berlin et al., 2000, S. 3-20

[Reich/Stucki 2002]

Reich, F., Stucki, T., Definition eines Geschäftsmodells für die MIGROSBANK als Grundlage für ein Multichannel-Management, in: Leist, S., Winter, R., Retail Banking im Informationszeitalter - Integrierte Gestaltung der Geschäfts-, Prozess- und Applikationsebene, Springer, Berlin et al., 2002, S. 109-122

[Rüegg-Stürm 2000]

Rüegg-Stürm, J., Was ,ist' eine Unternehmung? Ein Unternehmungsmodell zur Einführung in die Grundkategorien einer modernen Managementlehre, Diskussionsbeiträge des Instituts für Betriebswirtschaftslehre Nr. 36, Universität St. Gallen, St. Gallen, 2000

[Rummler/Brache 1995]

Rummler, G.A., Brache A.P., Improving Performance, $2^{\text {nd }}$ ed., Jossey-Bass, San Francisco, 1995

[Scheer 1998]

Scheer, A.W., ARIS - Vom Geschäftsprozess zum Anwendungssystem, 3. Aufl., Springer, Berlin et al., 1998

[Schütte 1998]

Schütte, R., Grundsätze ordnungsmässiger Referenzmodellierung: Konstruktion konfigurations- und anpassungsorientierter Modelle, Gabler, Wiesbaden, 1998

[Sinz 1988]

Sinz, E.J., Das Strukturierte Entity-Relationship Modell (SERM), Angewandte Informatik, 30 (5), 1988), S. 191-202

[Turowski 2002]

Turowski, K. (Hrsg.), Vereinheitlichte Spezifikation von Fachkomponenten, Memorandum des AK 5.10.3 Komponentenbasierte betriebliche Anwendungssysteme, Universität Augsburg, 2002

[Winter 1996]

Winter, R., Towards an Integration of Structured Techniques for Data Modeling and Function Modeling in Information Systems Development, in: Dias Coelho, J. et al. (Eds.), Proc. 4th European Conference on Information Systems, Lissabon, 1996, S. 1003-1010

[Winter 1998]

Winter, R., Informationsableitung in betrieblichen Anwendungssystemen, Vieweg, Braunschweig/Wiesbaden, 1998 
[Winter 2000]

Winter, R., Zur Positionierung und Weiterentwicklung des Data Warehousing in der betrieblichen Applikationsarchitektur, in: Schmidt, H. (Hrsg.), Modellierung betrieblicher Informationssysteme (Proc. der MobIS-Fachtagung 2000), Rundbrief der GIFachgruppe 5.10, 7 (1), 2000, S. 23-38

[Winter 2002]

Winter, R., Retail Banking im Informationszeitalter, in: Leist, S., Winter, R., Retail Banking im Informationszeitalter - Integrierte Gestaltung der Geschäfts-, Prozess- und Applikationsebene, Springer, Berlin et al., 2002, S. 29-50

[Yourdon 1989]

Yourdon, E., Modern Structured Analysis, Prentice-Hall, Englewood Cliffs et al., 1989 [Yourdon/Constantine 1979]

Yourdon, E., Constantine L.L., Structured Design: Fundamentals of a Discipline of Computer Program and Systems Design, Prentice-Hall, Englewood Cliffs, 1979 